\title{
Terrain-Induced Turbulence over Lantau Island: 7 June 1994 Tropical Storm Russ Case Study
}

\author{
Terry l. Clark, Teddie Keller, Janice Coen, Peter Neilley, Hsiao-ming Hsu, and William D. Hall \\ National Center for Atmospheric Research, * Boulder, Colorado
}

(Manuscript received 13 August 1996, in final form 7 January 1997)

\begin{abstract}
Numerical simulations of terrain-induced turbulence associated with airflow over Lantau Island of Hong Kong are presented. Lantau is a relatively small island with three narrow peaks rising to between 700 and $950 \mathrm{~m}$ above mean sea level. This research was undertaken as part of a project to better understand and predict the nature of turbulence and shear at the new airport site on the island of Chek Lap Kok, which is located to the lee of Lantau. Intensive ground and aerial observations were taken from May through June 1994, during the Lantau Experiment (LANTEX). This paper focuses on flow associated with the passage of Tropical Storm Russ on 7 June 1994, during which severe turbulence was observed.

The nature of the environmental and topographic forcing on 7 June 1994 resulted in the turbulence and shear being dominated by the combination of topographic effects and surface friction. High-resolution numerical simulations, initialized using local sounding data, were performed using the Clark model. The simulation results indicate that gravity-wave dynamics played a very minor role in the flow distortion and generation of turbulence. As a result of this flow regime, relatively high vertical and horizontal resolution was required to simulate the mechanically generated turbulence associated with Tropical Storm Russ.

Results are presented using a vertical resolution of $10 \mathrm{~m}$ near the surface and with horizontal resolutions of both 125 and $62.5 \mathrm{~m}$ over local, nested domains of about $13-24 \mathrm{~km}$ on a side. The $125-\mathrm{m}$ model resolution simulated highly distorted flow in the lee of Lantau, with streaks emanating downstream from regions of sharp orographic gradients. At this resolution the streaks were nearly steady in time. At the higher horizontal resolution of $62.5 \mathrm{~m}$ the streaks became unstable, resulting in eddies advecting downstream within a distorted streaky mean flow similar to the $125-\mathrm{m}$ resolution simulation. The temporally averaged fields changed little with the increase in resolution; however, there was a three- to fourfold increase in the temporal variability of the flow, as indicated by the standard deviation of the wind from a 10-min temporal average. Overall, the higher resolution simulations compared quite well with the observations, whereas the lower resolution cases did not. The high-resolution experiments also showed a much broader horizontal and vertical extent for the transient eddies. The depth of orographic influence increased from about $200 \mathrm{~m}$ to over $600 \mathrm{~m}$ with the increase in resolution. A physical explanation, using simple linear arguments based on the blocking effects of the eddies, is presented. The nature of the flow separation is analyzed using Bernoulli's energy form to display the geometry of the separation bubbles. The height of the $80 \mathrm{~m}^{2} \mathrm{~s}^{-2}$ energy surface shows eddies forming in regions of large orographic gradients and advecting downstream.

Tests using both buoyancy excitation and stochastic backscatter to parameterize the underresolved dynamics at the $125-\mathrm{m}$ resolution are presented, as well as one experiment testing the influence of static stability suppressing turbulence development. All these tests showed no significant effect. Implications of these results to the parameterization of mechanically induced turbulence in complex terrain are discussed.
\end{abstract}

\section{Introduction}

The National Center for Atmospheric Research (NCAR) and the Hong Kong University of Science and Technology, in conjunction with the Royal Observatory of Hong Kong, conducted a modeling and observational

*The National Center for Atmospheric Research is sponsored by the National Science Foundation.

Corresponding author address: Dr. Terry L. Clark, NCAR/MMM, P.O. Box 3000, Boulder, CO 80307-3000.

E-mail: clark@ncar.ucar.edu study called LANTEX (Lantau Experiment). The main goal was to improve our understanding and prediction of terrain-induced windshear and turbulence (TIWT) at the site of a new airport in Hong Kong. The new airport is located on the small island of Chek Lap Kok (CLK), which, after construction, has an elevation of approximately $6 \mathrm{~m}$ above mean sea level (MSL) and lies immediately north of the mountainous Lantau Island. Lantau is nearly $20 \times 10 \mathrm{~km}$ with several peaks rising to about $900 \mathrm{~m}$ MSL. As the prevailing low-level wind in Hong Kong is generally from the east-southeast, the new airport site on CLK is frequently directly downstream of Lantau. Two distinct mechanisms causing the turbulence were identified, namely, gravity wave-critical- 
level flow interaction and mechanically generated (or frictionally induced) turbulence associated with high speed flow over Lantau Island. Under appropriate conditions, gravity waves excited by flow over Lantau Island interact with the environmental critical level, causing TIWT near Chek Lap Kok. In other cases, tropical storms with high wind speeds and deep uniform flow appear to produce mostly mechanically generated turbulence. We use the term "deep uniform flow" to refer to cases where the dynamical effects associated with wave trapping and critical levels can be ignored.

As part of LANTEX, the NCAR King Air conducted regular flights in the Hong Kong region from May to November 1994. During a 2-month Intensive Operational Period (IOP) (May and June 1994), the King Air data were supplemented by an extensive ground-based observing network that included a $2-\mu \mathrm{m}$ Doppler lidar, a network of automatic weather stations, a profiler on Lantau Island, and an upstream sounding system that included a second wind profiler. At least one severe and a number of moderate turbulence events were observed during the IOP. The present paper focuses on the predominantly mechanically generated turbulence produced by the $10-20 \mathrm{~m} \mathrm{~s}^{-1}$ deep uniform flow associated with the passage of Tropical Storm Russ on 7 June 1994. In the future, we plan to publish our findings on a case study of the moderate turbulence observed on 20 May 1994 resulting from critical-level flow, as well as on results from idealized critical-level flow.

Severe turbulence is frequently encountered in the lee of complex topography. Probably the first detailed aircraft observations of a terrain-induced windstorm were by Lilly and Zipser (1972) for the 11 January 1972 severe downslope windstorm near Boulder, Colorado. These observations inspired extensive research on the mechanisms responsible for such high-drag, strong surface wind flows. Using two-dimensional dynamics with free-slip lower boundary conditions, Clark and Peltier (1977), Klemp and Lilly (1978), Peltier and Clark (1979), Lilly and Klemp (1979), Durran and Klemp (1983), and Bacmeister and Pierrehumbert (1988), among others, helped establish the relative importance of various mechanisms in producing such windstorms. After some debate, it has become recognized that gravity-wave breaking plays a crucial role in these windstorms.

The analyses of Peltier and Clark (1983) and Clark and Peltier (1984) showed that the super-adiabatic region provided by wave overturning constructively reflects wave energy back to the surface, leading to highly amplified flow regimes. Defining $U$ as the mean flow speed, $N$ as the Brunt-Väisälä frequency, and $h$ as the mountain height, the latter paper showed that, for Froude numbers $(\mathrm{Fr}=U / N h)$ slightly larger than that required to produce wave overturning in deep uniform flow, the nonlinear critical-level flow resulted in highamplitude resonant response when the height of the critical level matched the heights of maximum streamline steepening for the deep uniform flow. These early works pointed to the concept that wave breaking acts as a selfinduced critical level, albeit a nonlinear critical level. Subsequent papers by Laprise and Peltier (1989) and Scinocca and Peltier (1991), among others, refined these concepts. The parameter space where these theories apply most readily is high, wide two-dimensional ridges such as the Front Range of the Colorado Rockies. Lantau Island is not only narrow, $N a / U \approx 1$ (where $a$ is the mountain half-width), causing the flow to be critically nonhydrostatic, but it is also highly three-dimensional, which further increases wave dispersion effects. Gravity waves obtained in the simulations were typically either low-amplitude vertically propagating waves excited by the forcing of the full horizontal extent of Lantau Island or somewhat higher amplitude evanescent modes excited by individual mountain peaks. Idealized deep uniform flow simulations using a range of wind speeds with $N \approx 0.01$ also showed no high-amplitude wave dynamics, although vortices were generated for low Froude number cases. Here, $N$ is effectively quite close to zero for tropical storms because of cloud processes. Thus, the high wind speeds, low static stability, narrowness, low height, and three-dimensionality of Lantau Island all combine to create a flow regime where we can expect mechanical turbulence to dominate as the source of TIWT during the passage of tropical storms. It is possible that $N a / U$ can be of $O(1)$, which is large enough that stability effects can still modify mechanical turbulence and cannot be entirely neglected. However, since we expect the solutions to be dominated by mechanical turbulence, substantial computing resources were expended on high-resolution simulations in order to capture flow separation and surface instabilities associated with the generation of mechanical turbulence.

Linear analytic solutions of turbulent flow over topography began with Jackson and Hunt's (1975) application of rapid-distortion theory [see Savill (1987) for an informative review of Batchelor and Townsend's rapid distortion theory] for boundary layer flow over small hills. Their two-dimensional solutions showed a significant speedup of the flow near the crest of the hill in accord with potential-flow theory. Mason and Sykes (1979) extended this theory to three dimensions. A number of field experiments were conducted to test Jackson and Hunt's theory. Two of these were confined to relatively low hills (e.g., Mason 1986; Mason and King 1985) where the measurements validated the expected speedup over the crest of the hill. Jenkins et al. (1981) measured the flow over the relatively steep, 330-m high island of Ailsa Craig, and found not only a speedup of the flow around the sides and over the summit of the island, but also a deceleration of the flow downstream and a powerful trailing vortex whose axis was aligned in the direction of the upstream wind. The flow was near neutral and relatively high speed $\left(10-20 \mathrm{~m} \mathrm{~s}^{-1}\right)$, limiting the source of the turbulence to mechanical. Balloon measurements of turbulence along the sides of the island 
indicated very high turbulence levels with a substantial increase, by about a factor of 6 , in the amplitude of the crosswind perturbation component as compared with the along-wind component. It is interesting to note that severe turbulence prevented gathering balloon data in the lee of this relatively small island. The observations from Ailsa Craig highlight the fact that the applicability of the linear theories is limited to small hills, that is, situations where finite amplitude and flow separation effects can both be ignored.

Many of the water tank experiments on flow over orography have concentrated on low Froude number effects such as upstream blocking (e.g., Baines 1979). However, for Froude numbers greater than one, and for cases where $N a / U \gg 1$, flow separation can occur, resulting in a large recirculation region on the leeward slope (Hunt and Snyder 1980). In wind tunnel experiments for neutral flow, large flow fluctuations are evident and intermittent horseshoe vortices may exist (Hunt and Snyder 1980). Comparison between wind tunnel and water tank experiments elucidated the effects of stratification. For example, for triangular-shaped obstacles, Castro et al. (1983) found that stratification caused separation of the upstream boundary layer to depend much more on hill aspect ratio than in the neutral case. They found that the presence of gravity waves could significantly affect the size of the separation zone behind the obstacle, with the magnitude of the effect depending on the gravity wave vertical wavelengths.

Baines and Manins (1989) performed water tank experiments using the actual topography of Lantau Island for cases with $\mathrm{Fr}<1$, which is too small a Froude number to apply to the conditions during the passage of Tropical Storm Russ. Furthermore, it appears difficult to correlate results from these tank experiments with realistic conditions in the lee of Lantau Island. For example, none of the observations taken during LANTEX during weak flow conditions (i.e., for similar ranges of Fr) indicated the presence of strong aircraft turbulence. The fact that the amplitudes of the perturbation flow in the tank experiments were large compared with the mean flow speeds does not necessarily imply strong turbulence as Baines and Manins suggest.

Numerical simulations of turbulent flow over topography have focused primarily on simulating neutral boundary-layer flow over small hills. Ayotte et al. (1994) evaluated the impact of six different turbulence closure schemes on the mean flow and turbulent quantities using the mixed spectral finite-difference model. Their results were restricted to linear flow situations with small terrain slopes, that is, with no separation in the lee of the hill. Xu et al. (1994) investigated turbulence closure schemes using the same model but for both linear and nonlinear calculations, and found that the closure schemes could impact the predictions of form drag. However, they did not address the mechanisms responsible for generation of the turbulence. The linear, three-dimensional simulations of Ayotte and Tay- lor (1995) showed the expected acceleration of the flow over the hill, associated with the convergence of streamlines and accompanying negative pressure perturbation. Note that these simulations cannot be directly compared with the present work as they lack both the important finite amplitude effects of the mountain and flow separation effects.

The two-dimensional simulations of Romero et al. (1995) of hydrostatic flow over a bell-shaped mountain with flow speeds of $20 \mathrm{~m} \mathrm{~s}^{-1}$ showed the turbulence level was sensitive to the atmospheric stability. They found that the turbulence intensity increased with decreasing stability, and their moist simulation produced more turbulence than their dry simulation, presumably because the static stability decreased around midday.

Our study of Tropical Storm Russ concentrates on ascertaining the influence of both horizontal resolution and static stability on the simulated turbulence over Lantau Island. Only the highest resolution simulation (using 62.5-m horizontal grid spacing) is compared with observations, as it is only at this resolution that the simulations show turbulence characteristics realistic enough to allow comparison. As will be shown, resolution influences the simulations of terrain-induced turbulence dramatically where, until one looks closely, one sees what might be termed a resolution bifurcation between the $125-\mathrm{m}$ and $62.5-\mathrm{m}$ horizontal resolution cases. We present physical explanations of these dramatic developments, which also have some bearing on the subject of parameterization of surface stress for larger scale models. Experiments on the sensitivity to variations in static stability were performed at the lower horizontal resolution of $125 \mathrm{~m}$ and, as such, require some care in interpretation.

The paper is organized as follows. Section 2 describes the model and experimental design. Section 3 presents lower resolution experiments showing the influence of static stability on the terrain-induced turbulence. Sensitivity experiments on the formulation of subgrid-scale parameterizations are also discussed in this section. Section 4 presents the highest resolution results and a comparison with the coarse grid results. Section 5 compares the high-resolution simulations with the observations. Section 6 concludes the paper.

\section{Model description and experimental design}

These calculations exploit the two-way interactive nesting and grid refinement features of the model described by Clark (1977), Clark and Farley (1984), and Clark and Hall (1991, 1996), using four domains for the highest resolution case and three domains for the remaining cases. The outermost domain used 1000-m horizontal grid spacing and the three inner domains used grid spacing of 250, 125, and $62.5 \mathrm{~m}$, respectively. Figure 1a shows the orography $h(x, y)$ and outlines of all domains. Figure $1 \mathrm{~b}$ shows the orography of domains three and four, as well as the locations of surface weather 

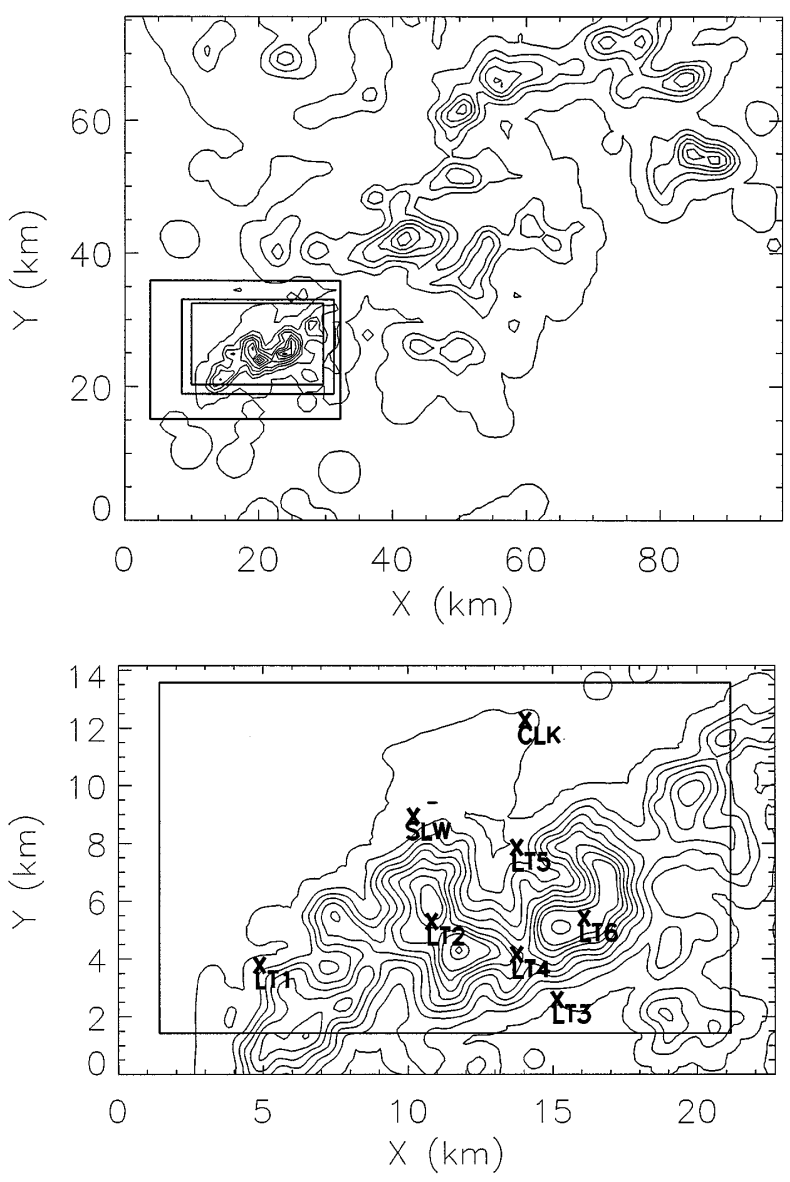

FIG. 1. Domain of integration. The full domain orography at a horizontal resolution of $1 \mathrm{~km}$ is shown in (a) and the third and fourth domains' orography and the location of automatic weather stations (AWS) are shown in (b). Locations of inner domains are shown in both panels. The contour interval is $90 \mathrm{~m}$ in both (a) and (b). stations. Inner domains were activated only after the flow in the parent domain had sufficient time to adjust to initialization. Other details of the experiments are described in Table 1.

\section{a. Vertical grid}

The vertical coordinate was transformed using

$$
z=F(\zeta)(1-h / H)+h,
$$

where $z$ is the Cartesian and $\zeta$ the transformed vertical coordinate. Here $F(\zeta)$ is defined such that $F(0)=0$ and $F(H)=\mathrm{H}$ where $H$ is the height of the outer domain. For the outer domain, a quadratic expansion in $\zeta$ was used near the surface. Letting $\zeta_{n}=(n-2) \Delta \zeta$, the value of $F(\zeta)$ at the $n$th grid level, $F(n)$, is taken as

$$
F(n)=27.25(n-2)+25.25(n-2)^{2}
$$

for the first nine levels. Then $F(n)$ was smoothly feathered into $\Delta z=500 \mathrm{~m}$ for the remaining troposphere. Note that the first $\zeta$ level is below the ground (i.e., $n$ $=1$ ) and the $n=2$ level is exactly at $z=h$. Using a hyperbolic tangent profile with a half-width of $1600 \mathrm{~m}$, a final stretching was applied near the tropopause until $\Delta z=750 \mathrm{~m}$ was achieved in the stratosphere. Three levels of vertical grid refinement across domains were used in these experiments with two 2:1 resolution changes in the vertical resulting in about 10 -m vertical resolution near the surface and $125 \mathrm{~m}$ aloft for domain three. The fourth domain used the same vertical grid structure as domain three. The inner grids are calculated using the vertical interpolation function of the model, which, as described by Clark and Hall (1996), is required to provide a consistent treatment of the Jacobian.

The present vertical grid was designed to give smooth variations in $\Delta z$ and $F_{\zeta \zeta^{*}}$. The latter is important because

\begin{tabular}{|c|c|c|c|c|c|c|c|c|c|}
\hline & Experiment & $\begin{array}{l}\text { No. } \\
\text { domains }\end{array}$ & $\begin{array}{l}(\Delta x)_{\min }, \\
\Delta S\end{array}$ & $\begin{array}{l}\text { Added } \\
\text { excitation }\end{array}$ & CSTK & $\begin{array}{l}\text { Environment } \\
\text { profile }\end{array}$ & $\begin{array}{l}\text { Simula- } \\
\text { tion } \\
\text { period } \\
\text { with } \\
(\Delta x)_{\min }\end{array}$ & $\mathrm{Ri}_{\mathrm{c}}$ & $\begin{array}{l}\text { Height } \\
\text { switch to } \\
\text { free atmos. } \\
\text { (inner } \\
\text { domain) }\end{array}$ \\
\hline 1 & HRUSSC & 3 & $125 \mathrm{~m}$ & None & 0.2 & sounding & $60 \mathrm{~min}$ & 0.0 & $62.5 \mathrm{~m}$ \\
\hline 2 & HRUSSE & 3 & $125 \mathrm{~m}$ & $\begin{array}{l}\text { Buoy. pert } \\
< \pm 1 \mathrm{~K}\end{array}$ & 0.2 & sounding & $10 \mathrm{~min}$ & 0.0 & $62.5 \mathrm{~m}$ \\
\hline 3 & HRUSSE (cont.) & 3 & $125 \mathrm{~m}$ & $\begin{array}{l}\text { Buoy. pert } \\
< \pm 1 \mathrm{~K}\end{array}$ & 0.2 & sounding & $5 \mathrm{~min}$ & 0.0 & $125 \mathrm{~m}$ \\
\hline 4 & HRUSSE (cont.) & 3 & $125 \mathrm{~m}$ & $\begin{array}{l}\text { Buoy. pert } \\
< \pm 1 \mathrm{~K}\end{array}$ & $\begin{array}{l}0.1(z<125 \mathrm{~m}) \\
0.2(z \geq 125 \mathrm{~m})\end{array}$ & sounding & $45 \min$ & 0.0 & $125 \mathrm{~m}$ \\
\hline 5 & HRUSSB & 3 & $125 \mathrm{~m}$ & $\begin{array}{l}\text { Noise applied } \\
\text { to } \mathrm{K}_{\mathrm{m}}\end{array}$ & $\begin{array}{l}0.1(z<125 \mathrm{~m}) \\
0.2(z \geq 125 \mathrm{~m})\end{array}$ & sounding & $60 \min$ & 0.0 & $125 \mathrm{~m}$ \\
\hline 6 & HR4M & 4 & $62.5 \mathrm{~m}$ & $\begin{array}{l}\text { Buoy. pert } \\
< \pm 1 \mathrm{~K}\end{array}$ & $\begin{array}{l}0.1(z<125 \mathrm{~m}) \\
0.2(z \geq 125 \mathrm{~m})\end{array}$ & sounding & $60 \mathrm{~min}$ & 0.0 & $62.5 \mathrm{~m}$ \\
\hline 7 & HRUSSN & 3 & $125 \mathrm{~m}$ & None & 0.2 & $\begin{array}{l}\text { Winds from sounding, } \\
\text { Neutral static stability }\end{array}$ & $60 \min$ & 0.0 & $62.5 \mathrm{~m}$ \\
\hline 8 & HRUSSN (cont.) & 3 & $125 \mathrm{~m}$ & $\begin{array}{l}\text { Buoy. pert } \\
\quad< \pm 1 \mathrm{~K}\end{array}$ & 0.2 & $\begin{array}{l}\text { Winds from sounding, } \\
\text { Neutral static stability }\end{array}$ & $10 \mathrm{~min}$ & 0.0 & $125 \mathrm{~m}$ \\
\hline 9 & HRUSSN (cont.) & 3 & $125 \mathrm{~m}$ & $\begin{array}{l}\text { Buoy. pert } \\
< \pm 1 \mathrm{~K}\end{array}$ & 0.2 & $\begin{array}{l}\text { Winds from sounding, } \\
\text { Neutral static stability }\end{array}$ & $5 \mathrm{~min}$ & 0.1 & $125 \mathrm{~m}$ \\
\hline
\end{tabular}

TABLE 1. Details of numerical experiments. 
sharp gradients in $F_{\zeta \zeta}$ can generate noise through the diagnostic pressure equation (Clark and Hall 1996). Prior to our recognizing the importance of designing smooth grids, calculations produced rather noisy patterns in the dynamics with spikes in the second derivatives of the coordinate transformation. The present grid is sufficiently smooth so that this model artifact is avoided and vertical filtering was not necessary. Figure 2 shows $\Delta z$ and $F_{\zeta \zeta}$ versus $z$ for the three levels of vertical grid refinement used in the experiments. Some low-amplitude noise, which had no detectable effect on the calculations, is seen in $F_{\zeta \zeta}$ for the third level of refinement.

\section{b. Governing equations}

Explicit treatment of moisture is left to a later study, as moist processes are neglected in the present simulations. We expect moisture would act to reduce the effective static stability and modestly increase turbulence intensities, and this effect is explored in section 3 by varying $N$. Long- and shortwave radiation and Coriolis effects were also neglected due to the small horizontal extent of the domain. It was also assumed that sensible heat flux over Lantau would have a negligible effect on the overall dynamics in the present flow regimes (Reisner and Smolarkiewicz 1994).

The model treats the transformed Navier-Stokes equations as

$$
\begin{aligned}
\bar{\rho} \frac{\partial u}{\partial t}+ & \nabla \cdot(\bar{\rho} \mathbf{V} u) \\
= & -\left(\frac{\partial}{\partial x} \sqrt{J} p+\frac{\partial}{\partial \zeta} \sqrt{J} J^{13} p\right)+\frac{\partial}{\partial x}\left(\sqrt{J} \tau_{11}\right) \\
& +\frac{\partial}{\partial y}\left(\sqrt{J} \tau_{12}\right)+\frac{\partial}{\partial \zeta}\left(\sqrt{J} J^{13} \tau_{11}+\sqrt{J} J^{23} \tau_{12}+\tau_{13}\right),
\end{aligned}
$$

$$
\begin{aligned}
\bar{\rho} \frac{\partial v}{\partial t}+ & \nabla \cdot(\bar{\rho} \mathbf{V} v) \\
= & -\left(\frac{\partial}{\partial y} \sqrt{J} p+\frac{\partial}{\partial \zeta} \sqrt{J} J^{23} p\right)+\frac{\partial}{\partial x}\left(\sqrt{J} \tau_{21}\right) \\
& +\frac{\partial}{\partial y}\left(\sqrt{J} \tau_{22}\right)+\frac{\partial}{\partial \zeta}\left(\sqrt{J} J^{13} \tau_{21}+\sqrt{J} J^{23} \tau_{22}+\tau_{23}\right),
\end{aligned}
$$

$$
\begin{aligned}
\bar{\rho} \frac{\partial w}{\partial t} & +\boldsymbol{\nabla} \cdot(\bar{\rho} \mathbf{V} w) \\
= & -\rho_{0} \frac{\partial}{\partial \zeta}\left(p / \rho_{0}\right)+\bar{\rho} g B+\frac{\partial}{\partial x}\left(\sqrt{J} \tau_{31}\right) \\
& +\frac{\partial}{\partial y}\left(\sqrt{ } \bar{J} \tau_{32}\right)+\frac{\partial}{\partial \zeta}\left(\sqrt{J} J^{13} \tau_{31}+\sqrt{J} J^{23} \tau_{32}+\tau_{33}\right)
\end{aligned}
$$

where $u, v$, and $w$ are the Cartesian components of the air velocity in the $x, y$, and $z$ directions, respectively. Here, $\mathbf{V}$ is the transformed advection velocity where $\mathbf{V}$ $=u \mathbf{i}+v \mathbf{j}+\omega \zeta$ where $\omega$ is the velocity component normal to $\zeta$ surfaces and is given as

$$
\sqrt{J} \omega=w+\sqrt{J} J^{13} u+\sqrt{J} J^{23} v
$$

and in the anelastic system the mass continuity equation takes the form

$$
\boldsymbol{\nabla} \cdot(\bar{\rho} \mathbf{V})=0 .
$$

In this framework, $\boldsymbol{\nabla}$ is defined as $(\partial / \partial x) \mathbf{i}+(\partial / \partial y) \mathbf{j}+$ $(\partial / \partial \zeta) \zeta$, and $\bar{\rho}=F_{\zeta} \rho_{0}(z)$ is the Jacobian weighted density where $\rho_{0}(z)$ is the reference dry air density. The perturbation pressure $p$ and buoyancy $B$ are treated in (5) equivalent to the Lipps and Hemler (1982) anelastic equations. The metric terms are given as

$$
\begin{aligned}
\sqrt{J} & =F_{\zeta}(1-h / H) \\
\sqrt{J} J^{13} & =\left(\frac{F(\zeta)}{H}-1\right) h_{x} \\
\sqrt{J} J^{23} & =\left(\frac{F(\zeta)}{H}-1\right) h_{y} .
\end{aligned}
$$

The Reynolds stress terms $\tau_{i j}$ are defined as

$$
\tau_{i j}=\rho_{0} K_{m} D_{i j},
$$

where $D_{i j}$ is the deformation tensor and $K_{m}$ is the coefficient of eddy mixing. The subgrid closure is taken from Smagorinsky (1963) and Lilly (1962) where

$$
\begin{aligned}
& K_{m} \\
& = \begin{cases}\left(\Delta_{l}\right)^{2}|\operatorname{Def}| \sqrt{1-\operatorname{Pr} \cdot \mathrm{Ri}} & \text { for } \mathrm{Ri}<\mathrm{Ri}_{\mathrm{c}} \text { and } z>\Delta_{\infty} \\
0 & \text { for } \mathrm{Ri}>\mathrm{Ri}_{\mathrm{c}} \text { and } z>\Delta_{\infty} \\
\left(\Delta_{l}\right)^{2}|\operatorname{Def}| & z<\Delta_{\infty},\end{cases}
\end{aligned}
$$

where $\mathrm{Ri}$ is the Richardson number and $\mathrm{Ri}_{\mathrm{c}}$ is a critical value (Table 1). The eddy Prandtl number (Pr) is set to unity giving $K_{m}=K_{\theta}$ where $K_{\theta}$ is the coefficient of eddy mixing for heat. The grid scale $\Delta_{l}$ used the Blackadar (1962) formulation

$$
\Delta_{l}=\kappa \frac{\left(z+z_{0}\right)}{1+\kappa\left(z+z_{0}\right) / \Delta_{\infty}},
$$

with $\Delta_{\infty}=.2 \Delta_{h}$ where $\Delta_{h}$ is the horizontal grid resolution and $k=.4$ is the von Kármán constant. Over land, $z_{0}$ was taken as $30 \mathrm{~cm}$, while free-slip conditions were used over the ocean. The deformation Def is given as

$$
\operatorname{Def}^{2}=\frac{1}{2} \sum_{i} \sum_{j} D_{i j}^{2} .
$$

The potential temperature $\theta$ is treated using the equaion 

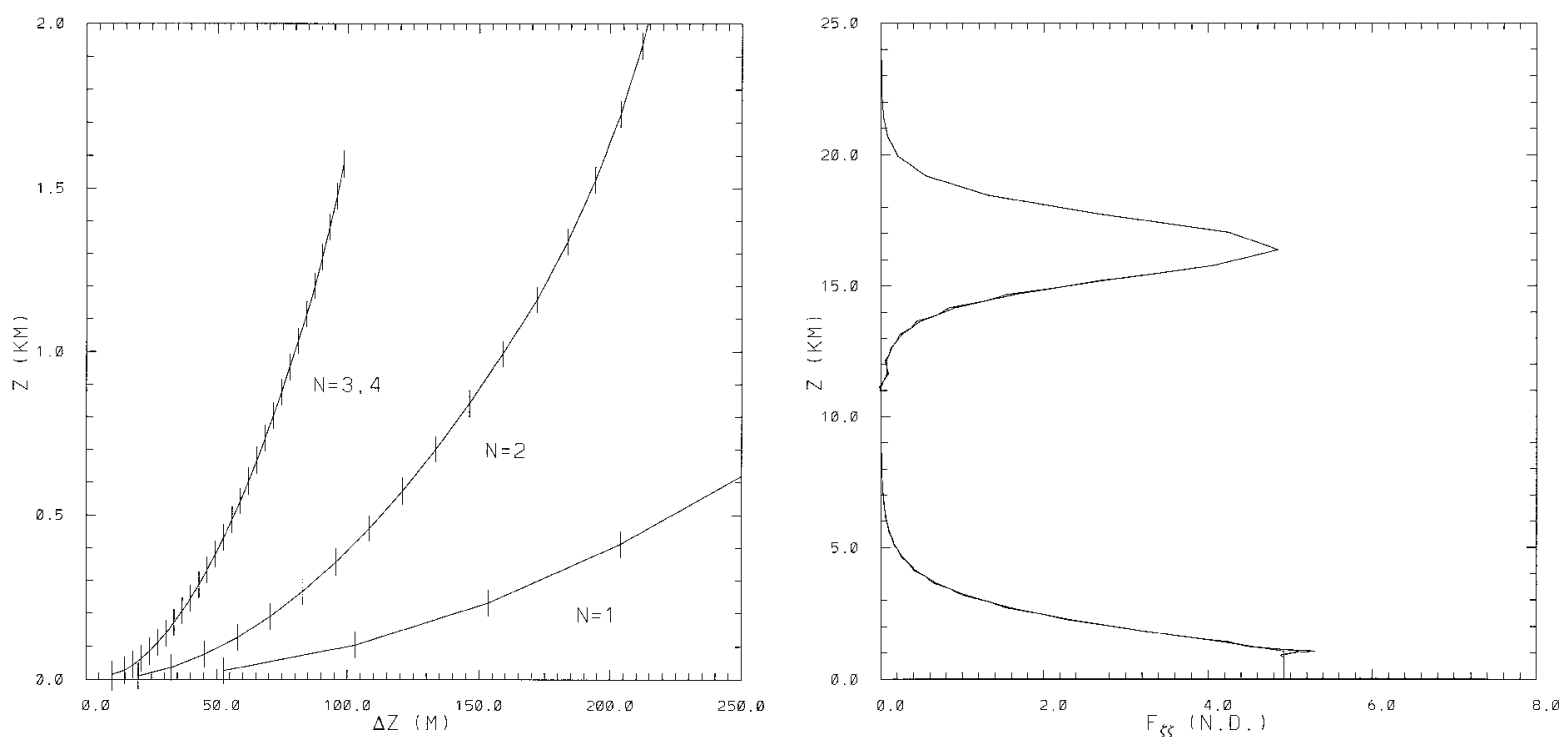

FIG. 2. Vertical grid structure. Grid size $\Delta z$ versus $z$ for the lowest $2 \mathrm{~km}$ is shown in (a) for the three levels of refinement and $F_{\zeta \zeta}$ is shown in (b).

$$
\begin{aligned}
\bar{\rho} \frac{\partial \theta}{\partial t}+ & \nabla \cdot(\bar{\rho} \mathbf{V} \theta) \\
= & +\frac{\partial}{\partial x}\left(\frac{\bar{\rho} K_{\theta}}{\sqrt{J}} \frac{\partial}{\partial x} \sqrt{J} \theta\right)+\frac{\partial}{\partial y}\left(\frac{\bar{\rho} K_{\theta}}{\sqrt{J}} \frac{\partial}{\partial y} \sqrt{J} \theta\right) \\
& +\frac{\partial}{\partial \zeta}\left(\frac{\bar{\rho} K_{\theta}}{\sqrt{J}} \frac{\partial}{\partial \zeta} \theta\right)+\frac{\partial}{\partial x}\left(\frac{\bar{\rho} K_{\theta}}{\sqrt{J}} \frac{\partial}{\partial \zeta} \sqrt{J} J^{13} \theta\right) \\
& +\frac{\partial}{\partial y}\left(\frac{\bar{\rho} K_{\theta}}{\sqrt{J}} \frac{\partial}{\partial \zeta} \sqrt{J} J^{23} \theta\right)
\end{aligned}
$$

and $B$ in (5) is treated as

$$
B=\frac{\theta-\bar{\theta}}{\bar{\theta}}
$$

where $\bar{\theta}$ is taken as a cubic least-squares fit to the ambient environment through the full model depth, that is, from $z=0$ to $H$.

The equations are approximated with second-order finite differences using the Arakawa (1966) quadratically conservative form for the advection of momentum and the Smolarkiewicz $(1983,1984)$ scheme for $\theta$.

Two measures used to analyze the model data were the cube root of the turbulent kinetic energy dissipation rate, $(\epsilon / \rho)^{1 / 3}$, and the temporal standard deviation of the wind speed, $\sigma_{q}$. The derivation of $\epsilon$ follows directly from the closure assumptions (Lilly 1962) and is defined as

$$
\epsilon=\tau_{i j} \frac{\partial u_{i}}{\partial x_{j}}=\rho_{0} K_{m}|\operatorname{Def}|^{2},
$$

where $u_{i}=(u, v, w)$. The turbulent eddy dissipation is a measure of the subgrid-scale energy or turbulence in the model data and can be directly compared with at- mospheric values. Values of $(\epsilon / \rho)^{1 / 3}>5 \mathrm{cgs}$ are typically associated with moderate turbulence, while values of $\approx 10 \mathrm{cgs}$ indicate severe turbulence in large aircraft.

To assess the level of resolved scale variability of the air motions, the temporal variance of the flow was calculated as

$$
\sigma_{u_{i}}^{2}(\mathbf{x}, t)=\left(u_{i}(\mathbf{x}, t)-\frac{1}{n} \sum_{t_{i}=t-(n / 2) \Delta t}^{t_{i}=t+(n / 2) \Delta t} u_{i}\left(\mathbf{x}, t_{i}\right)\right)^{2},
$$

where $\Delta t=10 \mathrm{~min}$ was used in (16) to analyze the present results. The standard deviation of the total variance $\sigma_{q}$, defined as

$$
\sigma_{q}=\sqrt{\left(\sigma_{u}^{2}+\sigma_{v}^{2}+\sigma_{w}^{2}\right)}
$$

is used to assess temporal variance of the wind field. It is important to recognize the difference between $(\epsilon / \rho)^{1 / 3}$ and $\sigma_{q}$. Large values of $\sigma_{q}$ do not necessarily imply large values of $(\epsilon / \rho)^{1 / 3}$, unless the fields comprising $\sigma_{q}$ are highly nonlinear and involve strong energy transfer to the smaller scales.

\section{c. Initial conditions}

The King Air flight data showed that the passage of Tropical Storm Russ on 6-8 June 1994 marked a significant terrain-induced turbulence event. Figure 3 shows the upstream sounding from Waglan Island, taken at 1300 local time on 7 June 1994, which was used in the simulations of Tropical Storm Russ. Waglan Island lies about $35 \mathrm{~km}$ east of Lantau Island and was the upstream sounding site during LANTEX. The raw sounding data were filtered to eliminate most of the high frequency variations in the data, particularly for wind speed and the static stability. The ambient conditions 

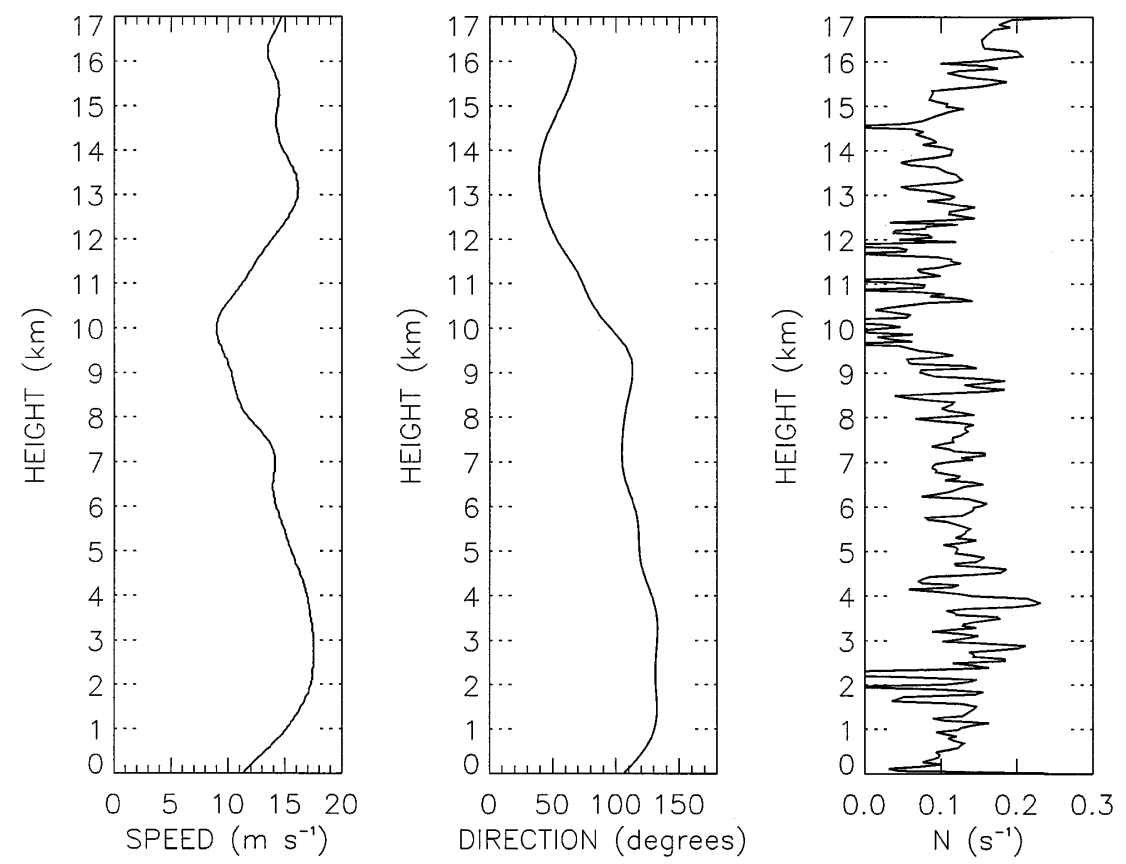

FIG. 3. Upstream soundings for Tropical Storm Russ taken at 1257 local time on 7 June 1994 showing wind speed (a), wind direction (b), and $N$ (c).

during this event were characterized by relatively uniform profiles of strong wind and stability over much of the troposphere. The wind was southeasterly at about $11-16 \mathrm{~m} \mathrm{~s}^{-1}$ in the first kilometer, eventually backing to the northeast above $300 \mathrm{mb}$. The low-level BruntVäisälä frequency $N$ was about $.013 \mathrm{~s}^{-1}$, assuming dry air.

The model was initialized using this single sounding, assuming horizontally uniform conditions in $x$ and $y$ at the model inflow boundaries. The winds and temperature were projected onto the model grid at the initial time. Mass continuity was ensured by adding a small perturbation potential flow component to the profile winds.

\section{Simulations using low horizontal resolution}

Preliminary simulations used horizontal resolutions of $500 \mathrm{~m}$. At this resolution, the model produced vertically propagating gravity waves; however, the simulations indicated neither wave-breaking strong shears nor turbulence. Simulations using horizontal resolutions of $250 \mathrm{~m}$ showed much more spatial variability of the horizontal wind downstream of Lantau Island at levels below about $300 \mathrm{~m}$. The increase in low-level spatial variability of the flow with increased resolution supports the idea that the turbulence during this case was generated mechanically rather than by a gravity wave mechanism. The severe turbulence observed on this day was completely absent in these low-resolution simulations as the mechanical sources of the turbulence were inadequately resolved. Both the 500- and 250-m resolu- tion simulations produced surprisingly steady solutions, suggesting extremely low effective Reynolds numbers. It was only when the horizontal resolution was increased to $125 \mathrm{~m}$ and the vertical to $10 \mathrm{~m}$ that mechanical effects began to appear, although these solutions were still quite steady in time. Several simulations were performed using 125-m horizontal resolutions on the inner domain; one using the upstream sounding from Waglan (Fig. 3) without any added excitation, one with the winds from the sounding combined with neutral stability, and several with excitation added. These are discussed below. A further increase in horizontal resolution to $62.5 \mathrm{~m}$ resulted in a highly transient flow, as will be shown in section 4 .

\section{a. The reference simulation: HRUSSC}

The control experiment HRUSSC uses the wind and temperature profiles shown in Fig. 3 as initial and upstream boundary conditions with no added excitation. The results showed streaks of wind speed, vertical velocity, and vorticity extending downstream of Lantau, from near the surface up to about $300 \mathrm{~m}$ above ground level (AGL). Figure 4 shows 10-min temporal means of the $\left|\mathbf{V}_{h}\right|$ and $w$ fields at 5 and $111 \mathrm{~m}$ AGL from experiment HRUSSC. At the lowest level, streaks appear to emanate from the upstream mountain peaks, where flow blocking by the mountains combined with surface friction generates vorticity and a highly distorted flow. The streaks extend downstream more than $10 \mathrm{~km}$. At $111 \mathrm{~m} \mathrm{AGL}$, the pattern shows some larger-scale variations indicative of gravity waves. The plots of $w$ 

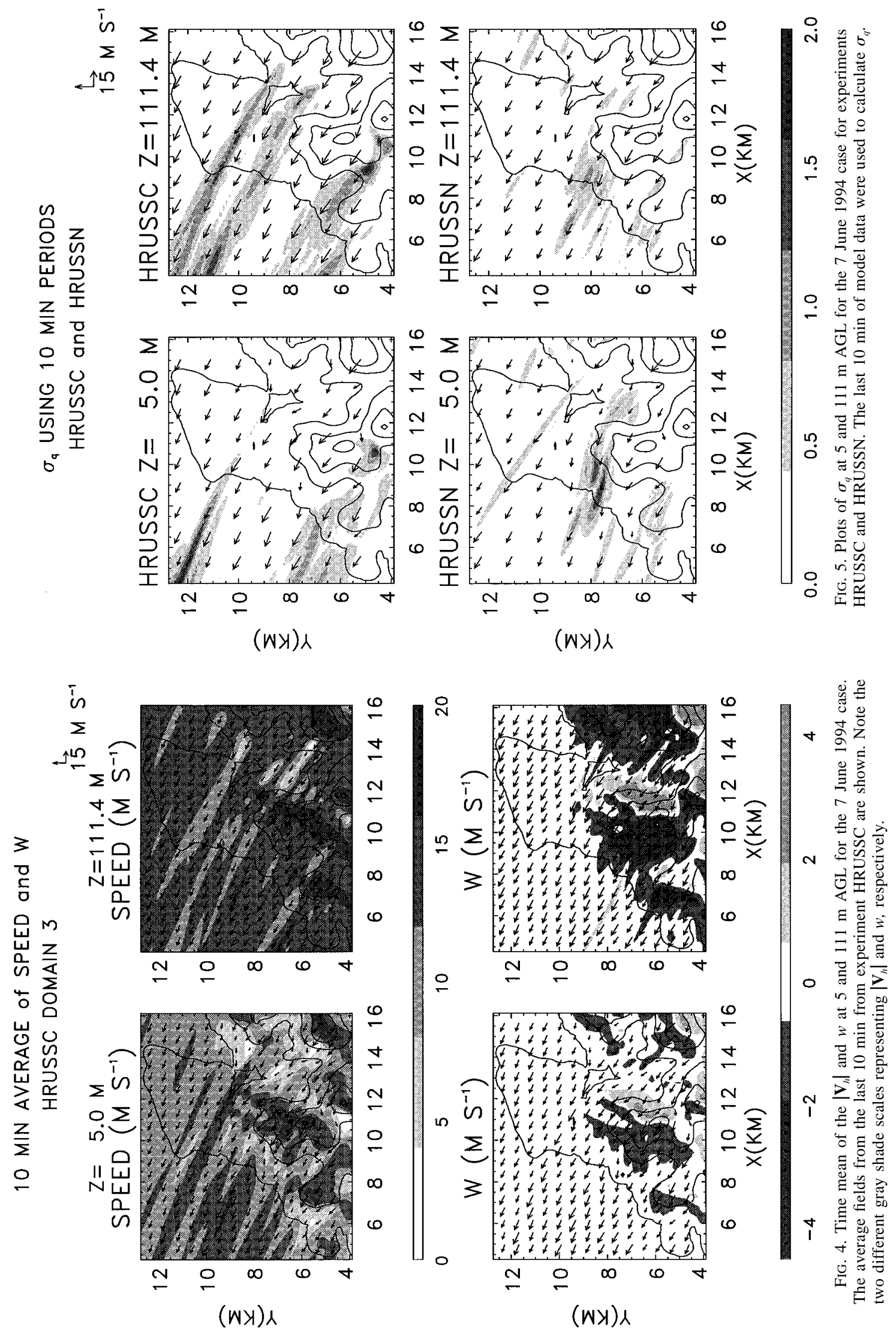
indicate persistent upwind updrafts over the mountain peaks with eddies and streaks extending downstream over CLK (see Fig. 1b for locations) and to the northwest. We see a strong return flow in the Tung Chung valley, which lies southeast of LT5 and east of LT2. Based on earlier experiments dominated by gravity wave dynamics (not shown), this return flow (or region of strong updraft) appears to result from a combination of forced ascent and gravity wave return flow in the lee of Sunset Peak (near LT6). As will be seen in section 4 , these temporally averaged fields show very little change between the $125-\mathrm{m}$ resolution control case (HRUSSC) and the 62.5-m (HR4M) case.

Figures $5 \mathrm{a}$ and $5 \mathrm{~b}$ show the temporal standard deviation of the wind speed $\sigma_{q}$ at the same two heights as in Fig. 4 for HRUSSC. Note that $\sigma_{q}$ is on the order of $1 \mathrm{~m} \mathrm{~s}^{-1}$ over the ocean remote from the peaks and $\approx 2$ $\mathrm{m} \mathrm{s}^{-1}$ near the peaks. Gray shading ranges from .5 to $2 \mathrm{~m} \mathrm{~s}^{-1}$ and all frames show only small areas with minor levels of temporal variability. This level of variability is small enough that individual snapshots of the fields are barely discernible from the temporally averaged fields.

As discussed earlier, a number of researchers have noted that increasing static stability suppresses the generation of mechanical turbulence (e.g., Castro et al. 1983; Romero et al. 1995). Thus the inclusion of cloud processes occurring on this day, which would act to decrease the stability, should increase the level of mechanical turbulence. To assess this effect, we reran HRUSSC with $N=0$ throughout the atmosphere, which should overestimate the effective reduction in static stability supplied by a moist atmosphere. However, putting $N=0$ does not address the effects of conditional instability provided by moist processes. We refer to this experiment as HRUSSN (see Table 1). The simulated wind patterns in HRUSSN were similar to those in HRUSSC, with streaks in wind speed and vorticity extending downstream from the mountain peaks. Figures $5 \mathrm{c}$ and $5 \mathrm{~d}$ show $\sigma_{q}$ from HRUSSN. We see a slight decrease in $\sigma_{q}$ for this case as compared to HRUSSC, which, as will be shown later, is probably due to the overall decrease in mean wind speeds for HRUSSN. HRUSSN has only $.5 \mathrm{~m} \mathrm{~s}^{-1}$ variability over the ocean, a similar maxima of $2 \mathrm{~m} \mathrm{~s}^{-1}$ near the peaks, but the position has changed somewhat compared to HRUSSC. As we will see later, these magnitudes of $\sigma_{q}$ are much smaller than observed by the aircraft on this day. These simulations suggest that the exclusion of moisture is not likely the reason for the model underestimating the observed levels of turbulence, since the effect of reduced stability has been overemphasized in experiment HRUSSN and shown to have little influence.

Except for their steadiness (in the low-resolution case), the low-level streaks obtained in these simulations appear similar to the flow in the lee of Ailsa Craig observed by Jenkins et al. (1981). They observed a powerful trailing vortex with its axis closely aligned with the upstream wind direction. Instead of the single peak (of $330 \mathrm{~m}$ ) for Ailsa Craig we have a number of peaks, three of them between 700 and $950 \mathrm{~m}$ in elevation, with about twice the width of Ailsa Craig.

\section{b. Effect of buoyancy and dynamic excitation}

Random noise is added to the model equations to either excite existing instabilities (e.g., Grabowski and Clark 1991) or to mimic physical effects such as stochastic backscatter (e.g., Leith 1990; Mason and Thomson 1992). Two experiments were performed to test the effects of excitation. Firstly, HRUSSC was continued for $1 \mathrm{~h}$ of simulation time, passively adding a $\pm 1 \mathrm{~K}$ white noise component to the buoyancy field only over land, and for $z<500 \mathrm{~m}$ AGL (called experiment HRUSSE). As in Grabowski and Clark (1991), the assumption is that the addition of this type of excitation will cause existing instabilities to develop more rapidly. Secondly, HRUSSE was continued for $1 \mathrm{~h}$ of simulation time, replacing the buoyancy excitation with dynamic excitation similar to that of Mason and Thompson (1992). This experiment was called HRUSSB, with "B" referring to backscatter. In experiment HRUSSB, we randomly modify the length scale $\Delta_{l}$ in (10) by premultiplying it with $c^{*}$, where $c^{*}$ is calculated using a random number generator based on the gamma distribution $P(y)$ $=4 y \exp (-2 y)$, which results in the mean value of $\bar{c}^{*}$ $=1$.

The results of both HRUSSE and HRUSSB show only very minor increases in the temporal and spatial variability of the flow resulting from excitation. Figure 6 shows $\sigma_{q}$ at 5 and $111 \mathrm{~m}$ AGL for HRUSSE and HRUSSB. These results show little change in the amplitude of $\sigma_{q}$ from those of HRUSSC shown in Fig. 5, which suggests that at this resolution there are few existing instabilities in the flow that may easily be excited. It is interesting to note that in this case buoyancy excitation leads to stronger variability, albeit small, than stochastic backscatter.

The role of stochastic backscatter is more to mimic the effects of a rough lower surface than to mimic the large-scale effects of topography. In his review paper, Finnigan (1988) points out that Schlichting's (1960) condition requires $u_{*} z_{0} / \nu>3$ to achieve Reynolds number independence of flow over a model hill in the wind tunnel. Estimates of $u_{*} z_{0} / \nu \approx 1$ were obtained for the present experiments by replacing $\nu$ with the value of $K_{M}$ at $5 \mathrm{~m} \mathrm{AGL}$ of the model. The small values of this parameter suggest we are treating Lantau Island as an aerodynamically smooth mountain, and that the distorted streaky flow is primarily the result of the local orography gradients affecting the viscous stress. In this case it seems reasonable to assume that sufficient horizontal resolution is necessary to resolve the distorted flow before realistic transient eddies can be simulated. 

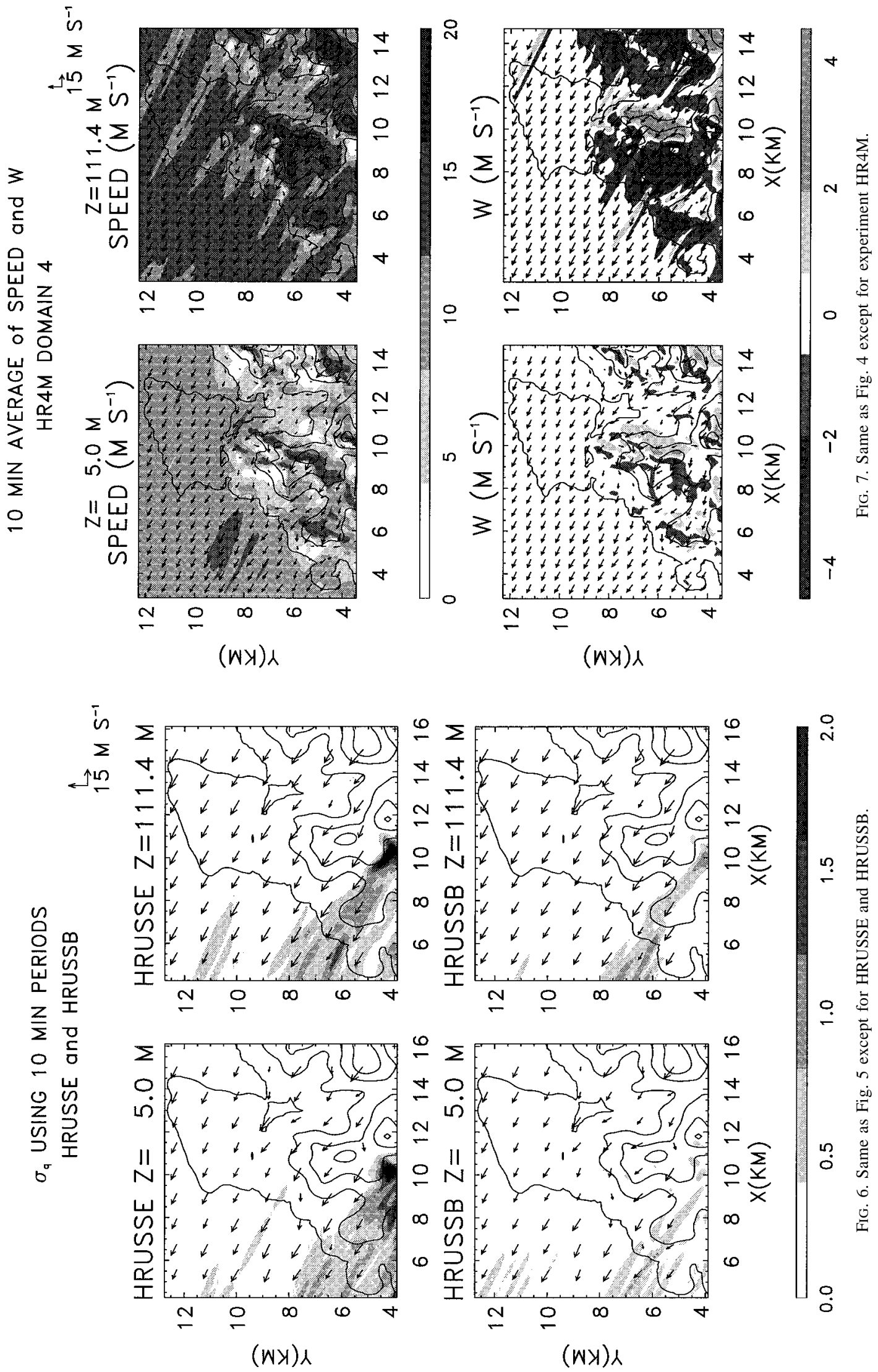


\section{$\sigma_{q}$ USING 10 MIN PERIODS \\ HR4M DOMAIN 4}

\section{$\stackrel{\vec{L}}{15 \mathrm{M} \mathrm{S}^{-1}}$}
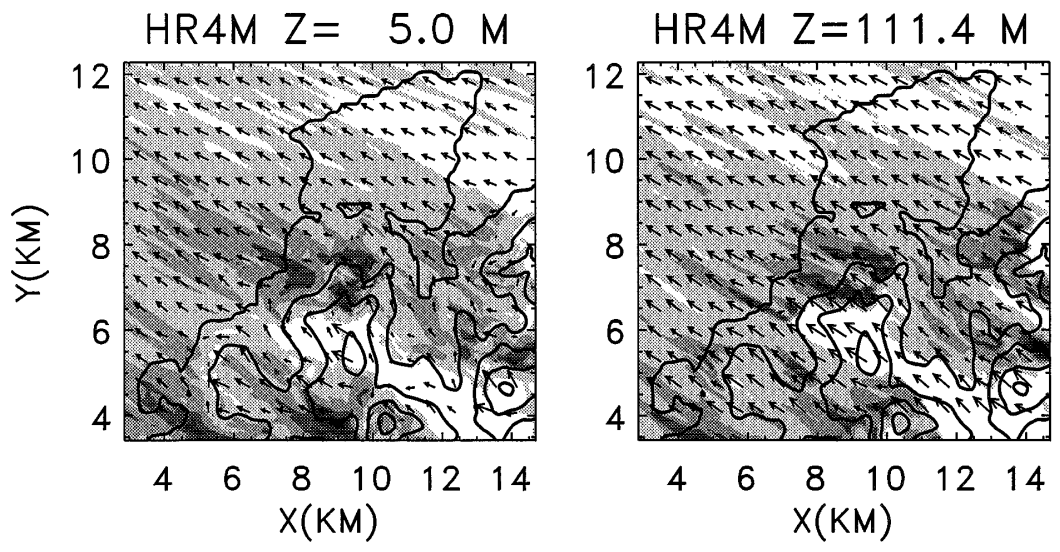

0

12

3

FIG. 8. Same as Fig. 5 except for HR4M. Note the gray shading range has doubled from that used in Fig. 5.

\section{Simulations using high horizontal resolution of $62.5 \mathrm{~m}$}

The steadiness of the streaks obtained using the 125-m horizontal resolution prompted us to consider using higher resolution to capture shearing instabilities associated with the distorted, local mean flow. Experi-
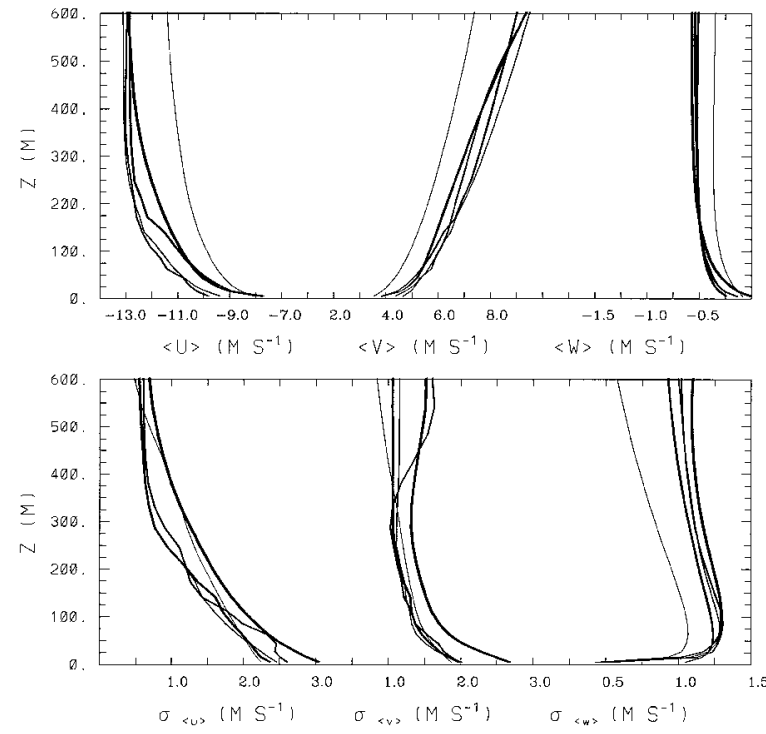

Fig. 9. Horizontal means and associated standard deviations of the temporally averaged velocity components. The area of averaging is identical to that displayed in Figs. 5-8. The thickest line shows the results of HR4M, whereas the thinnest line shows HRUSSN. The remaining lines, which are essentially equivalent to one another, represent HRUSSC, HRUSSE, and HRUSSB. ment HR4M is similar to HRUSSE, except that a fourth domain was added to locally increase the horizontal resolution from 125 to $62.5 \mathrm{~m}$. As Fig. 7 shows, this increase in horizontal resolution did not change the temporally averaged fields very much. However, the increase in resolution was sufficient, in this case, to effect a transition to turbulence. This is shown by the dramatic increase in the transient character of the flow (Fig. 8). In contrast to HRUSSC, which had values of about .5 $\mathrm{m} \mathrm{s}^{-1}$, experiment HR4M has a fourfold increase in $\sigma_{q}$ $\sim 2 \mathrm{~m} \mathrm{~s}^{-1}$ over the ocean as well as a significant increase in areal extent of the temporal variability. This is far more representative of the severe turbulence observed. These simulations are now realistic enough for comparison with the observations.

Before looking at the nature of the transient eddies, we present a summary of the statistics of the 125- and $62.5-\mathrm{m}$ results. Figure 9 shows the horizontal means and associated standard deviations of the temporally averaged velocity components. The area used in the averaging corresponds to that shown in Figs. 4-8.

As already discussed, the structure of the temporally averaged fields shows little change as a result of either excitation setting $N$ to zero or by doubling the horizontal resolution. Both the spatial mean \langle\rangle and standard deviation $\sigma_{\langle\rangle}$of temporally averaged $u, v$, and $w$ cluster about common vertical structures. Possibly the biggest difference seen in Fig. 9 is between HRUSSN and the other experiments, particularly for $\sigma_{\langle w\rangle}$. These results suggest that the source of temporal variability and subsequent turbulence is associated with instabilities occurring on the highly distorted flow. Analysis of these instabilities is a current topic of our research. 


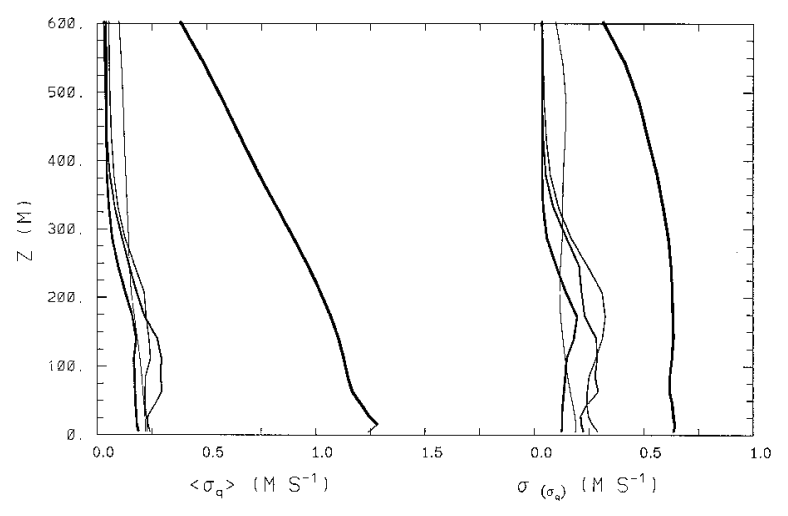

FIG. 10. Same as Fig. 9 except showing the horizontal means and standard deviations of $\sigma_{q}$.

Figure 10 shows the spatial mean of $\sigma_{q}$ and its associated standard deviation. Here we see a dramatic difference between HR4M $(62.5 \mathrm{~m})$ and the other $125-\mathrm{m}$ resolution experiments. The amplitudes of both $\left\langle\sigma_{q}\right\rangle$ and $\sigma_{\sigma_{q}}$ have increased by factors of about 4 and 3 , respectively. For the 125-m experiments, $\sigma_{q}$ has mostly decayed to an asymptotic value by $300 \mathrm{~m}$ AGL, whereas for HR4M the full depth of the high-resolution domain is filled with eddies whose amplitude slowly decrease with height above $300 \mathrm{~m}$ AGL. The next section presents a physical explanation for this dramatic change in amplitude and vertical structure of the eddies resulting from the increase in horizontal resolution. We do not expect similar differences to occur with further resolution doubling.

\section{a. Transient nature of the eddies and the separation bubble}

To help identify the nature of the mechanical turbulence in the high-resolution simulations, we estimate the envelope of the separation bubble using Bernoulli's energy form. Various values of

$$
Q_{B}=q^{2} / 2+p / \rho-g z \theta^{*}
$$

were plotted for both the 125- and 62.5-m horizontal resolution simulations. In (18), $q^{2}=u^{2}+v^{2}+w^{2}$ and $\theta^{*}=(\theta-\theta) / \theta$. We found that $Q_{B}=80 \mathrm{~m}^{2} \mathrm{~s}^{-2}$ gave a good representation of the flow that skimmed the surface just upstream of the mountains and outlined the regions of separation occurring downstream. Figures 11a and $11 \mathrm{~b}$ show two vertical cross sections from HRUSSB and HR4M, respectively, that are closely aligned with the mean wind direction. Figure 11a shows that for HRUSSB the $Q_{B}=80$ contour stays reasonably close to the mountain and departs significantly from the surface only over the water. Animation of this cross section shows this pattern is relatively steady. On the other hand, Fig. 11b shows significant departures of the $Q_{B}$ $=80$ contour, both over the mountain and downstream. Animation of these results shows a highly transient char-
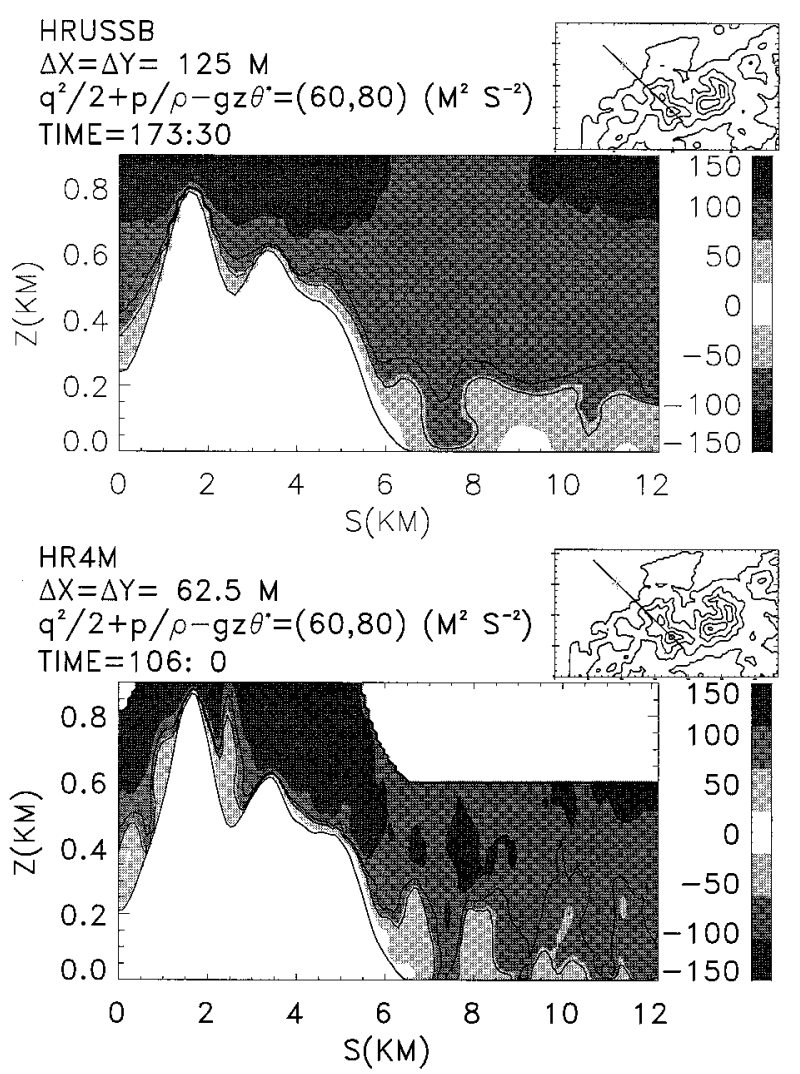

FIG. 11. Vertical cross sections of $Q_{B}$ for (a) HRUSSB at $t=173.5$ min and (b) HR4M at $t=105.75 \mathrm{~min}$. Contours of $Q_{B}=60$ and 80 $\mathrm{m}^{2} \mathrm{~s}^{-2}$ are shown. There is no negative shaded areas showing on the plot.

acter. Using a fixed value of $Q_{B}$ to display the separation bubble in three-dimensions assumes we are looking at regions of rapid distortion where viscous mixing has had little time to smooth out flow structures in the vertical. Otherwise, $Q_{B}$ would not approximate a conservative variable. We are also looking at values of $Q_{B}$ close enough to the surface so as to avoid multiple value problems in our selection of $Q_{B}$.

Having demonstrated the utility of near surface values of $Q_{B}$ giving an approximate representation of the separation bubble, the analysis is easily modified to show the full three-dimensional pattern of the flow separation. Figure 12 shows the height AGL of $Q_{B}=80$ for four times in experiment HR4M. The plot shows regularly spaced eddies propagating downstream of the mountain. The speed of propagation is about $17 \mathrm{~m} \mathrm{~s}^{-1}$, which corresponds to the mean flow speed. An animation of these fields shows regular patterns forming mostly in the lee of the peaks, as well as at the outflows of some of the valleys. After forming, the eddies propagate downstream and reform, repeating the cycle. The maximum height of the separation bubbles regularly exceeds the full depth of the fourth domain, which was $600 \mathrm{~m}$. The penetrations shown are then to some extent affected by the change in resolution occurring at this height level. 


$$
\begin{gathered}
\text { Height in } M \text { AGL of } \\
q^{2} / 2+p / \rho-g z \theta^{\circ}\left(M^{2} S^{-2}\right)=80 M^{2} S^{-2} \\
\text { HR4M DOMAIN } 4
\end{gathered}
$$
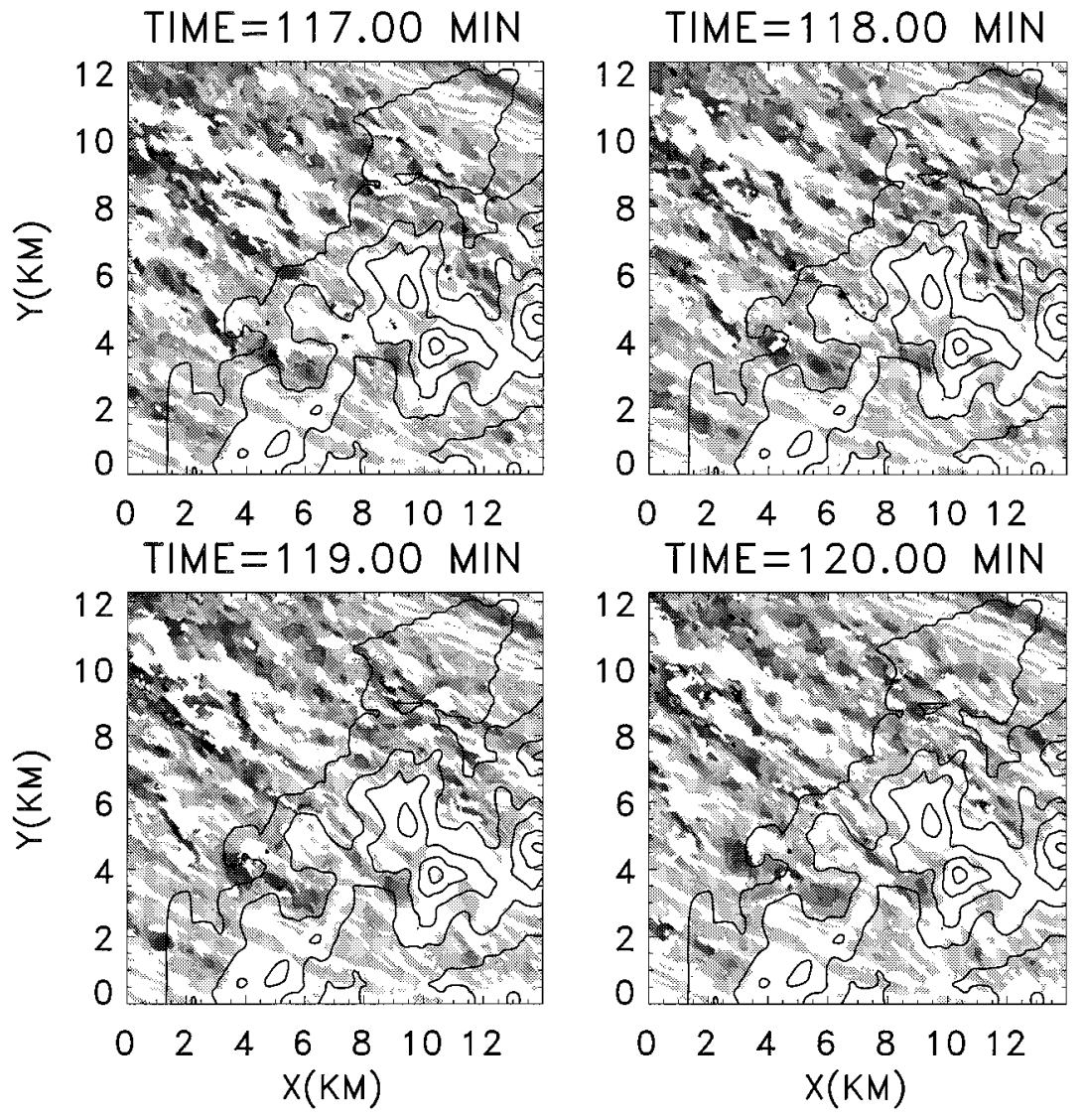

\begin{tabular}{lllllll}
\hline 0 & 100 & 200 & 300 & 400 & 500 & 600
\end{tabular}

FIG. 12. Horizontal plots of the height of $Q_{B}=80 \mathrm{~m}^{2} \mathrm{~s}^{-2}$ at four times for experiment HR4M.

The maximum 600-m penetration depth shown in Fig. 12 should therefore be viewed as an underestimate.

To understand the dramatic difference in results between HRUSSB and HR4M, we analyzed the near surface gradients of pressure and stress. Finnigan (1988) points out that it is important for a model to be able to reasonably represent $-\nabla_{h} p+\partial \tau / \partial z$ as $z \rightarrow h$. If, for example, similarity theory were applied giving $\partial \tau / \partial z=$ 0 , then the only term that can contribute to slowing down the air motion near the surface, in a manner to cause separation, is the adverse pressure gradient. We did not assume a constant flux layer in the present calculations, although it does appear that the adverse pressure gradients clearly dominate in causing the flow separation. The magnitude of $\partial \tau_{i 3} / \partial z$ is about $10-20$ times smaller than that of the adverse pressure gradients, and therefore it played a minor role in causing separation in these simulations. Figure 13 shows $-\nabla_{h} p$ for
HRUSSB and HR4M at $5 \mathrm{~m}$ AGL. We see that for HRUSSB the adverse pressure gradients are about -7 $\mathrm{mb} \mathrm{km}{ }^{-1}$ at the mountain peaks, whereas in HR4M the values more than double, at $-18 \mathrm{mb} \mathrm{km}^{-1}$. This increase in the adverse pressure gradients was sufficient to significantly enhance flow separation and, with the increased horizontal resolution, allowed the separation bubbles to form and propagate downstream.

\section{b. Vertical extent of the transient eddies}

A rough estimate of the horizontal spacing of the $Q_{B}$ surfaces using the correlation analysis

$$
\begin{aligned}
& c_{f}(\xi, \eta) \\
& \quad=\iint Q_{B}(x+\xi, y+\eta) Q_{B}(x, y) d x d y
\end{aligned}
$$


$-\vec{s} \cdot \nabla_{H} P(m b / K M) A T Z=5 . M A G L$ HRUSSB: MAX MIN $=8.15-6.88$ TIME $=173.50 \mathrm{MIN} \quad \mathrm{T} \mathrm{m} / \mathrm{s}$

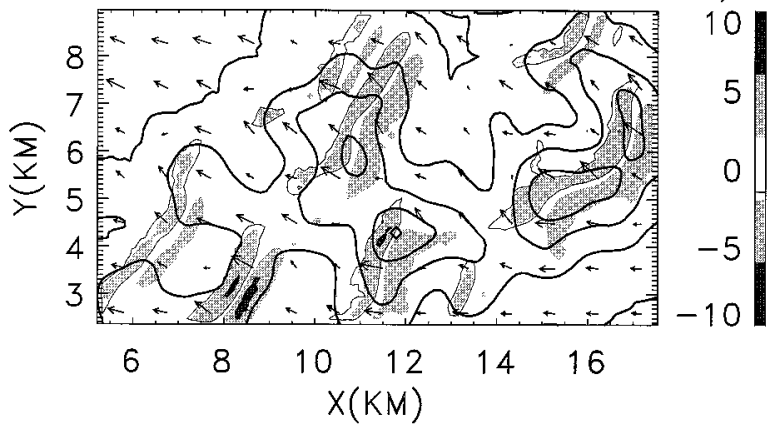

HR4M: MAX MIN $=19.14-18.03$ TIME $=105.75 \mathrm{MIN} \quad \overrightarrow{15} \mathrm{M} / \mathrm{s}$

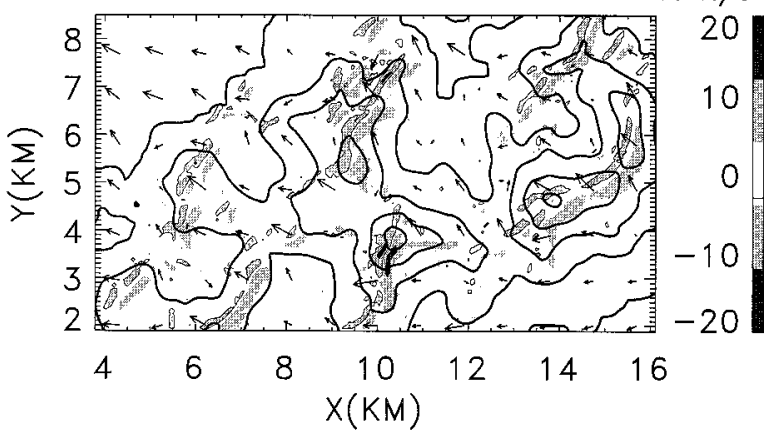

FIG. 13. Horizontal plots of $-\nabla_{h} p$ at $5 \mathrm{~m}$ AGL for (a) HRUSSB at $t=173.5 \mathrm{~min}$ and (b) HR4M at $t=105.75 \mathrm{~min}$. The units are $\mathrm{mb} \mathrm{km}{ }^{-1}$. Note the change in gray shading scale between (a) and (b).

showed two dominant scales at $\left(\lambda_{x}, \lambda_{y}\right)=(2.1,2.6)$ and $(3.5,3.5) \mathrm{km}$. The area of integration in (19) was confined to an area north of the peaks running through the center of the island. Figure 14 shows a plot of $c_{f}$ derived from taking an arithmetic mean of five different calculations of $c_{f}$ spaced 10-min apart. The two dominant scales are quite clear and visually correspond to the scale seen earlier in Fig. 12. Some interesting relations between the horizontal scale of the propagating eddies and their vertical structure can be understood through inspection of the steady-state wave equation,

$$
\hat{w}_{z z}+\left(-k^{2}-\frac{U_{z z}}{U}\right) \hat{w}=0,
$$

where we have ignored the contribution due to static stability and are assuming $U$ is the speed in the mean flow direction. If we assume a logarithmic profile to $U$ where

$U=U_{0}\left(1-e^{-\alpha z}\right)$, then $\frac{U_{z z}}{U}=-\alpha^{2} \frac{e^{-\alpha z}}{\left(1-e^{-\alpha z}\right)}$.

First we note that $-k^{2}-U_{z z} / U$ equals zero at

$$
z_{\ell}=-\frac{1}{\alpha} \ln \left(\frac{k^{2}}{\alpha^{2}+k^{2}}\right),
$$

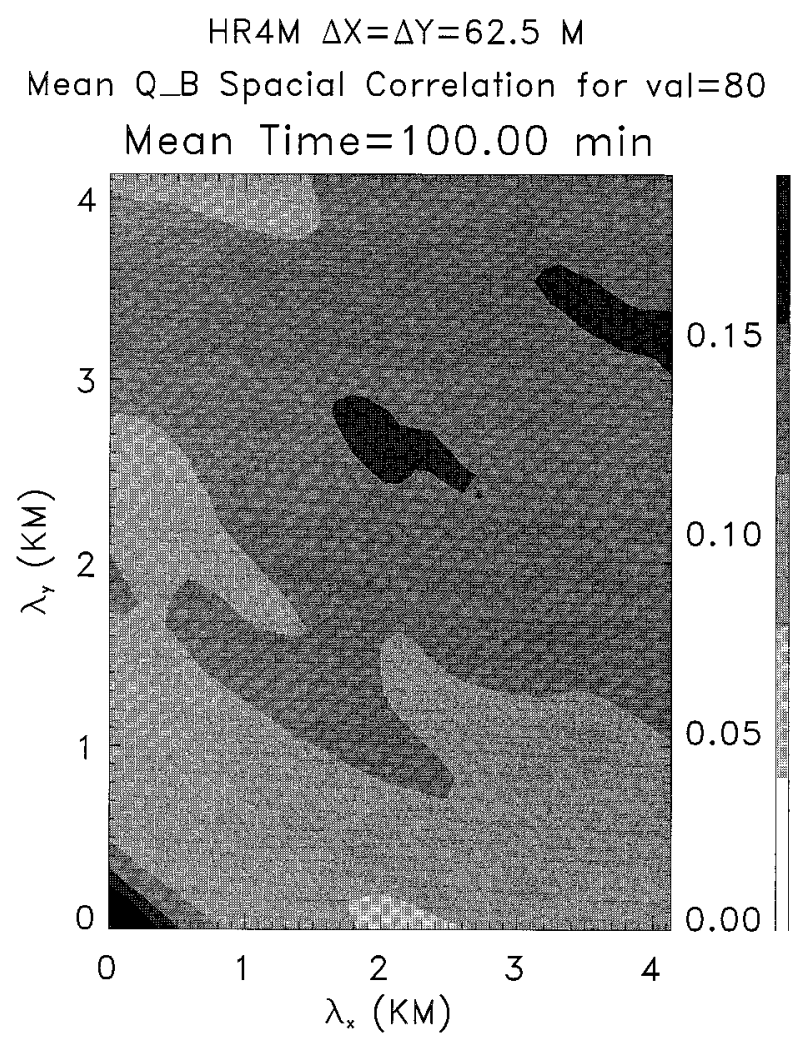

FIG. 14. Correlation analysis of the horizontal scale of eddies from HR4M. See text for details.

which is the level where the eddies change from being propagating to evanescent. For $1 / \alpha \sim 20 \mathrm{~m}$, we estimate $z_{l}$ is 45 and $50 \mathrm{~m}$ for the two dominant scales shown in Fig. 14. For $z \gg z_{l}$ we can ignore the curvature term and calculate the vertical scale of the eddies assuming they decay exponentially as $\exp (-k z)=\exp \left(-z / \lambda_{z}\right)$. Using this relation, the two correlation scales noted in Fig. 14 correspond to values of $\lambda_{z}=260$ and $393 \mathrm{~m}$. These depths are in good agreement with the results from the simulation shown in Figs. $11 \mathrm{~b}$ and 12.

It seems appropriate at this point to summarize our understanding of what caused the differences between the low- and high-resolution simulations of Tropical Storm Russ. In the low-resolution case, the effective viscosity of the distorted flow was too high to allow flow separation to induce vortex shedding. The lack of transient eddies provided no downstream disturbances that could cause local flow blocking and provide a vertical depth to the turbulence in accord with (20). On the other hand, the 62.5-m horizontal resolution in HR4M was sufficient to cause strong separation effects resulting in vortex shedding. The downstream propagation of these eddies in a sheared environment caused local drag similar to that which a small obstacle might provide in neutral flow. Another analogy is the excitation of deep vertical modes at the sheared interface at the top of the boundary layer caused by the penetration of rising ther- 


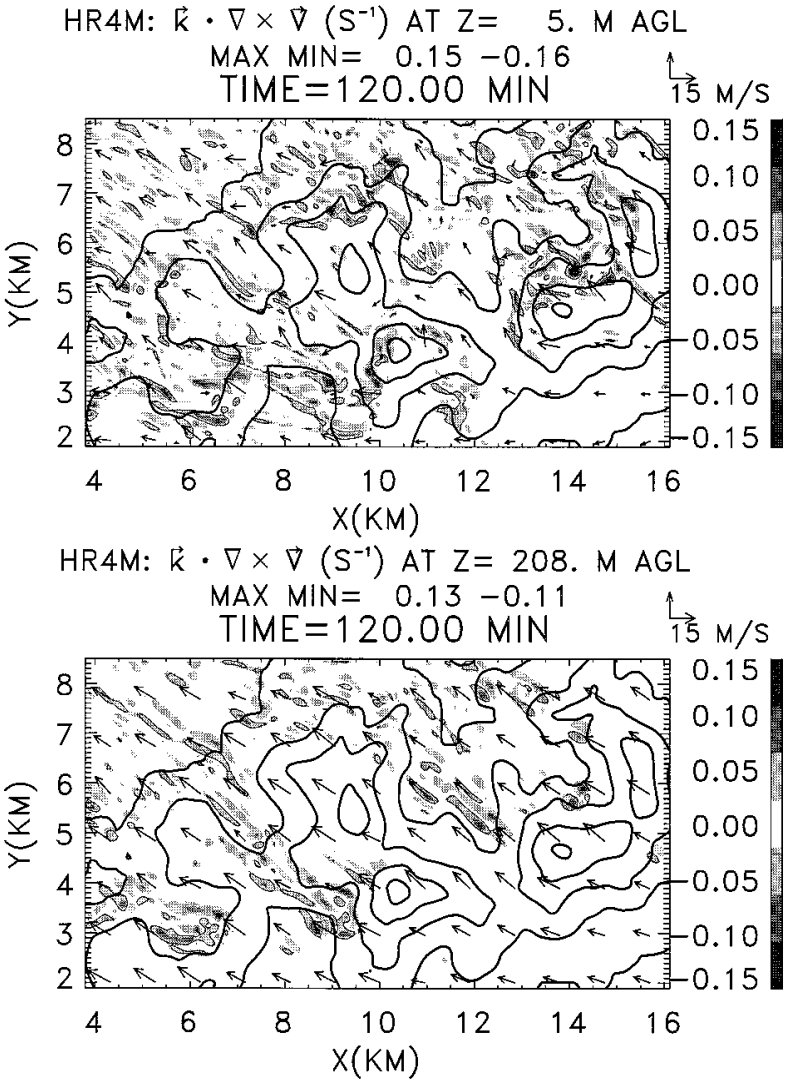

FIG. 15. Plan view of vertical vorticity at $t=120 \mathrm{~min}$ from $\mathrm{HR} 4 \mathrm{M}$ at $z=5$ and $208 \mathrm{~m} \mathrm{AGL}$.

mals in the thermal wave study of Clark et al. (1986). The vertical extent of the eddies scaled with the linear equations of motion, giving exponential decay heights of the eddies commensurate with their horizontal scales. This type of transition into a turbulence regime accounts for the rather dramatic differences caused by this increase in horizontal resolution from 125 to $62.5 \mathrm{~m}$. In the higher resolution simulation the model entered into the turbulence regime, resulting in what we are calling a resolution bifurcation.

As a final discussion on the character of the highresolution flow, Fig. 15 shows the vertical vorticity at 5 and $208 \mathrm{~m} \mathrm{AGL}$ for HR4M. The structure of the vortices at these heights is clear from the figure, where streamers of vorticity emanate from the irregular terrain. Animation of the results shows these structures are highly time dependent. Plots of $w$ and $|\mathbf{V}|$ were also analyzed and they do not show anywhere near the consistent trailing vortex structure evident in Fig. 15.

\section{Comparisons with observations}

In this section results of experiment HR4M are compared with observations collected during LANTEX. The $2-\mu \mathrm{m}$ lidar that was present during LANTEX was unfortunately not operating on 7 June. We must, then, confine our comparisons to surface anemometer and aircraft data.

\section{a. Surface weather stations}

Figure 16 shows a time-series comparison between the observed automatic weather station (AWS) data at AWS sites LT1 through LT6, CLK, and SLW (see Fig. 1b) between 0500 and 0600 UTC (or 1300 to 1400 local time) and the simulated data. The model data were taken every $15 \mathrm{~s}$ from the high-resolution domain of experiment HR4M interpolated to $10 \mathrm{~m}$ AGL. The observations were processed with a 1-min running mean filter on 1-s data.

The comparisons between model and observed wind speeds are overall quite good. Except for the mountaintop stations LT2 and LT6, the model simulations are within a few $\mathrm{m} \mathrm{s}^{-1}$ of the observations. A visual inspection indicates the variability in the model data is typically slightly less than that in the observations. At the high-elevation stations, LT6 and LT2, the model is underestimating the wind speed by 5 to $10 \mathrm{~m} \mathrm{~s}^{-1}$. It is important to note, however, that the simulations showed strong gradients in wind speed at the mountain peaks, which could easily account for these differences. The wind directions from the simulation compared extremely well with the observations, except for stations LT1 and LT2, where the model and observed directions show a difference of about $30^{\circ}$ and $50^{\circ}$, respectively. It is interesting that the errors in the model predicted winds show a northern bias at LT1 and an equal southern bias at LT2, suggesting a slight shift in the position of a propagating eddy. One noteworthy difference is that the observations show a stronger variability in both direction and speed than seen in the simulations.

\section{b. Aircraft}

Figure 17 shows a flight track and the winds observed by the NCAR King Air aircraft while flying at about $150 \mathrm{~m}$ AGL north of Lantau Island over the southern runway of CLK. The barbs in Fig. 17 represent 1-s averages from the $25-\mathrm{Hz}$ data and are shown every $5 \mathrm{~s}$. The winds are from the southeast at about 10 to $15 \mathrm{~m}$ $\mathrm{s}^{-1}$, and with significant variations along the flight track. For comparison, Fig. 18 shows five realizations of the model and the 1-min filtered aircraft data along the same flight track including wind speed, wind direction, and altitude. The comparisons are quite good, showing similar speeds and directions between the model and observations. A visual inspection indicates similar levels of variability between the observations and model, with wind speeds in the model ranging from 5 to $15 \mathrm{~m} \mathrm{~s}^{-1}$ and wind speed in the observations ranging from 10 to $15 \mathrm{~m} \mathrm{~s}^{-1}$. The model wind directions tend to be a little more southerly than the observations, by perhaps as much as $10^{\circ}$.

A comparison of $(\epsilon / \rho)^{1 / 3}$ in cgs units is also shown 


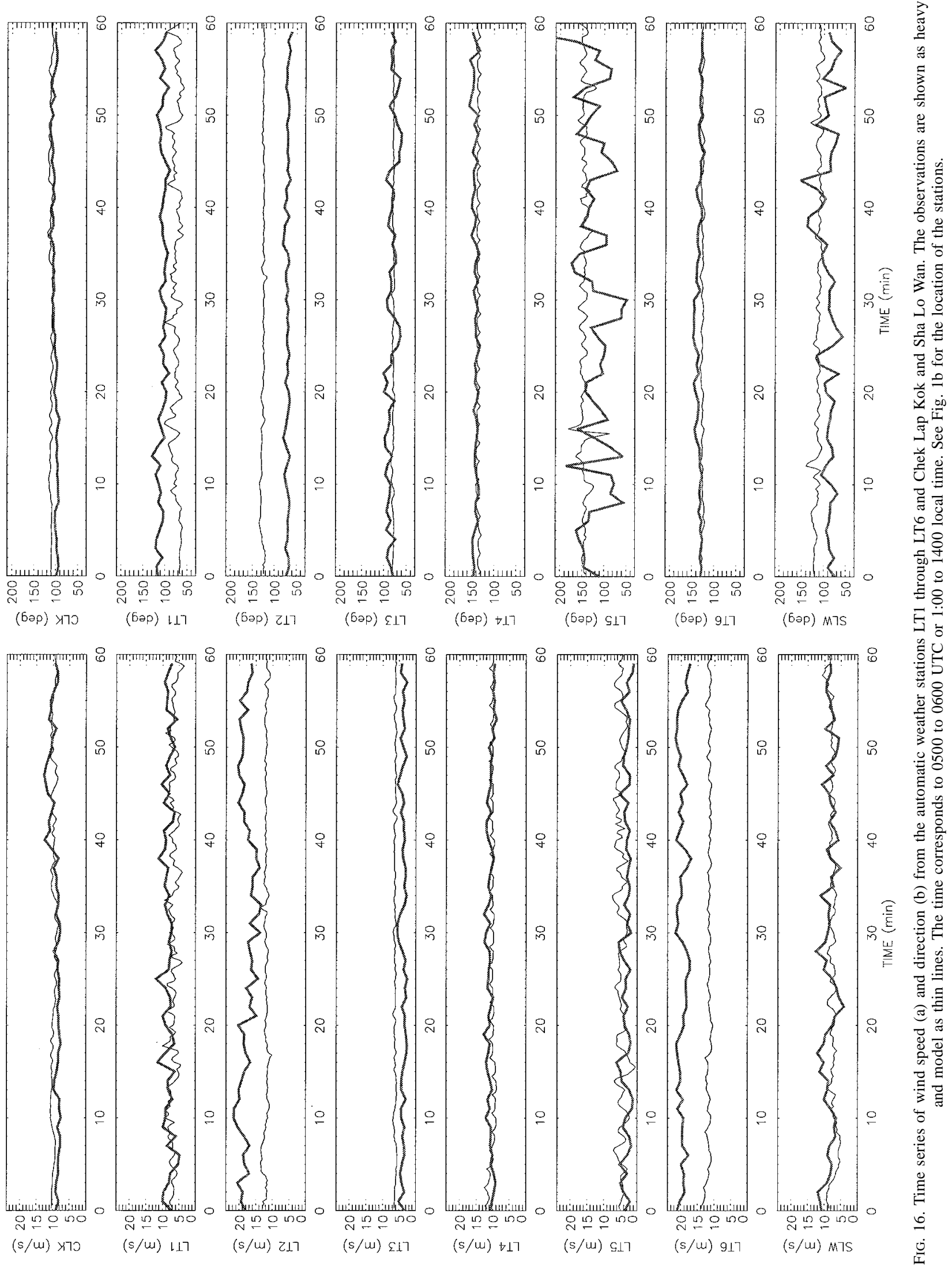




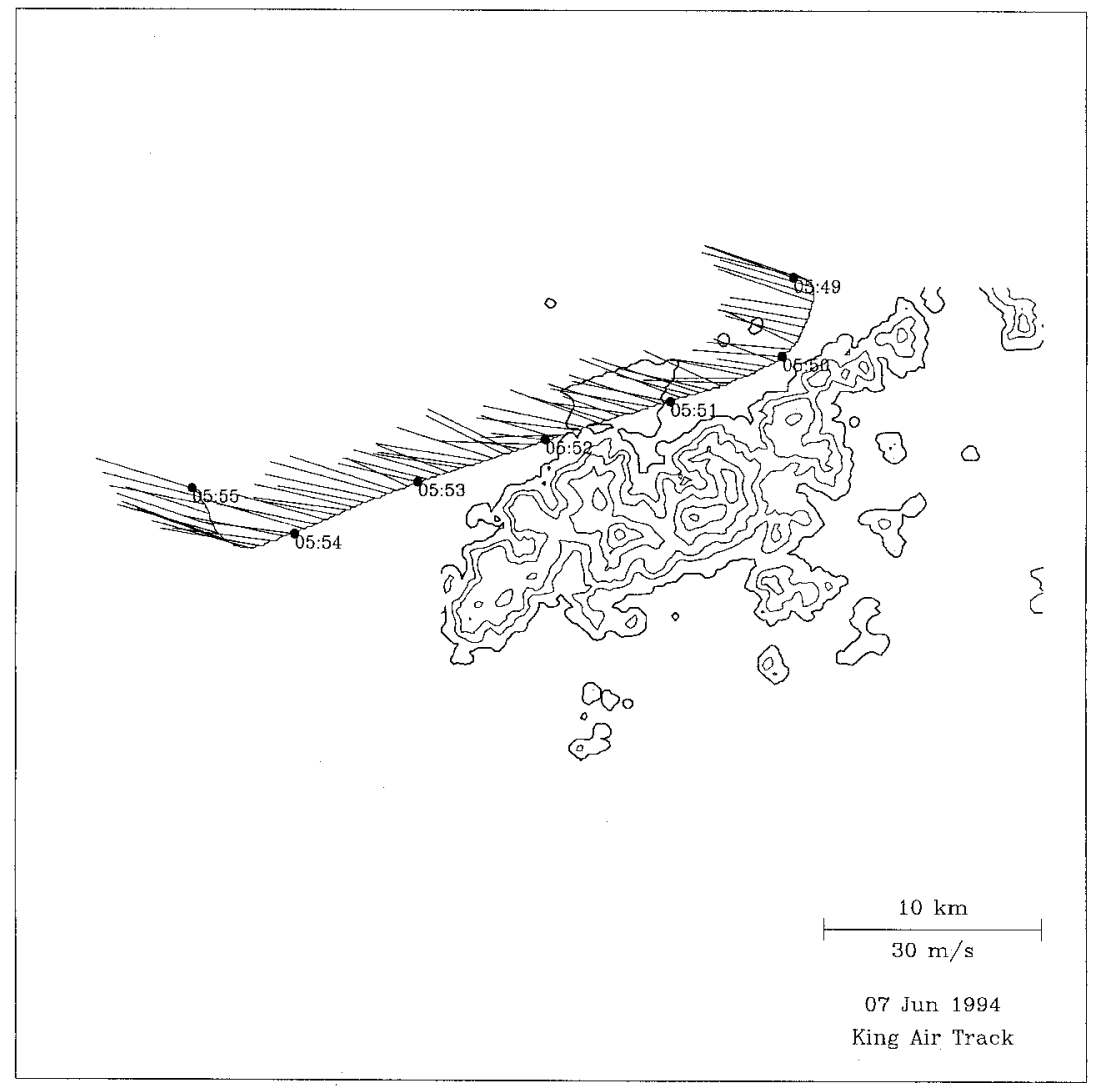

FIG. 17. Plan view of a flight track of the King Air on 7 June 1994. The wind barbs are plotted every $5 \mathrm{sec}$. The topography of Lantau and CLK are shown with contours of $1 \mathrm{~m}$, $200 \mathrm{~m}, 400 \mathrm{~m}, 600 \mathrm{~m}$, and $800 \mathrm{~m}$. The cross marks the start of the flight track.

in Fig. 18 for the same track. Both the data and model show values as high as 10 , which is representative of severe turbulence. Other tracks show a similar level of comparison between the model and observations for the high-resolution simulation. The fact that the aircraft data represent 10 -s temporal ( $\sim 1 \mathrm{~km}$ spatial) averages may explain most of the differences seen in the spatial variability of $(\epsilon / \rho)^{1 / 3}$ in Fig. 18.

\section{Conclusions}

Numerical simulations of the airflow over Lantau Island during the passage of Tropical Storm Russ (7 June 1994) were presented. The experiments used either 125 or $62.5-\mathrm{m}$ horizontal resolutions in the finest resolution domain, which covered areas of either $24 \times 15$ or 21 $\times 13 \mathrm{~km}$, respectively. These horizontal resolutions correspond roughly to either .1 or .05 times the half-widths of any of the three major peaks of Lantau, respectively. The vertical resolution was $10 \mathrm{~m}$ at the surface, which was smoothly stretched to $100 \mathrm{~m}$ aloft for these two inner domains. Sensitivity tests on the subgrid-scale parameterization were performed using the lower gridscale resolution. In one case, white noise perturbations were passively added to the buoyancy field, and in a second case, the stress terms were premultiplied with a random gamma distribution effecting a stochastic backscatter parameterization similar to that of Mason and Thompson (1992). One further simulation totally eliminated gravity waves by setting $N=0$ throughout the atmosphere, which was intended to test whether static stability was suppressing the development of mechanical turbulence.

All simulations showed very similar temporally averaged flow structures using a 10-min averaging period. Streaks of speed, vorticity, and vertical velocity streamed off the peaks of Lantau and propagated downstream. These streaks resulted from the combination of surface friction and topographic irregularities distorting the flow. Analysis showed that the surface was modeled as aerodynamically smooth, indicating that variations in the treatment of $z_{0}$ had only a minor effect. Analysis of the 125-m resolution simulation showed the streaks were surprisingly steady with time, whereas at $62.5-\mathrm{m}$ resolution the flow was highly transient. In the later case, the flow resembled that observed for Ailsa Craig by Jenkins et al. (1981), where a wake of turbulent, slowmoving flow straddled on both sides by turbulent high speed flow propagating downstream of the obstacles was found. Streaks have been observed by Smith et al. 


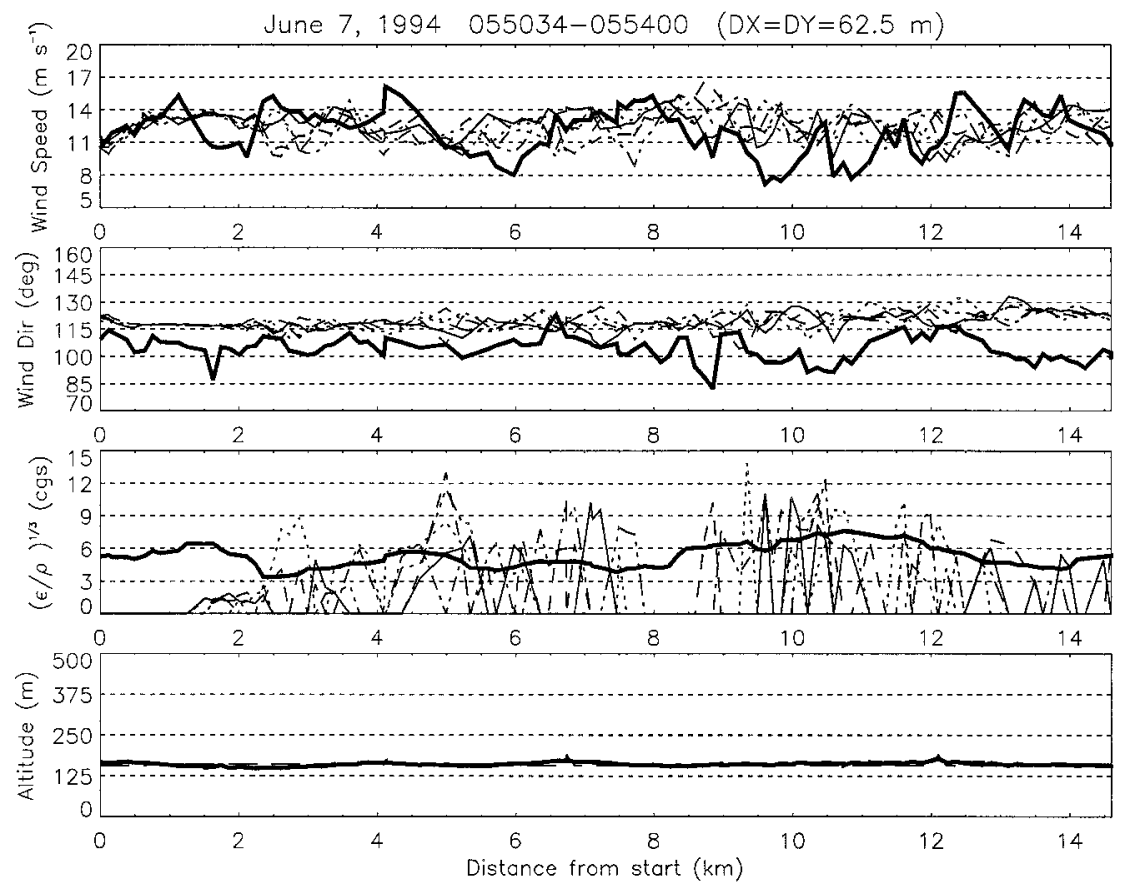

FIG. 18. Flight track data of wind speed, wind direction, elevation, and $(\epsilon / \rho)^{1 / 3}$ in cgs units. Five realizations from the model (thin lines) are shown with the observed data (thick line).

(1997) downstream of St. Vincent Island, using sun glint seen by satellite.

The experiments using both buoyancy excitation and stochastic backscatter at the $125-\mathrm{m}$ resolution showed that these processes had very little effect on the downstream turbulence. The experiment using $N=0$ also showed no significant effect. These results are in agreement with the concept that the turbulence is associated with topographic effects and has little to do with aerodynamic roughness. The results suggest that as the resolution is increased the flow distortions produced by the topography become unstable, resulting in the generation of transient eddies. It was also noted that the adverse pressure gradient approximately doubled from -7 to $-18 \mathrm{mb} \mathrm{km}^{-1}$ with the increase in resolution promoting vigorous flow separation in the model and vortex shedding at the $62.5-\mathrm{m}$ resolution. As a result of the onset of instabilities and/or more vigorous flow separation there was what we are calling a resolution bifurcation where the solutions went from near steady-state flow streamers below about $200 \mathrm{~m}$ AGL to a highly transient and turbulent flow with propagating eddies extending to at least $600 \mathrm{~m}$ AGL. Considering that this transition appears explicable in terms of simple physics, we do not expect similar dramatic changes with further increased resolution.

The nature of the turbulence was displayed using the Bernoulli energy form as an estimate of the envelope of the separation bubble. Viewing vertical cross sections, it was found that a near-surface value of the Bernoulli energy gave a useful approximation of the surface of flow separation. The main approximation is that the flow is rapidly distorted and the turbulence dissipates slowly downstream. This simple technique proved quite useful in showing eddies forming near strong gradients of orography aligned normal to the flow, which then propagate downstream. The vertical depth of penetration for some of these separation regions exceeded the inner domains height of $600 \mathrm{~m} \mathrm{AGL}$. Knowing this, future simulations will be designed with an appropriate increase in the vertical extent of the fine resolution domain. An analysis of the horizontal scale of the propagating eddies showed that their vertical depth is commensurate with that predicted by the linear dispersion relation. The transient eddies act as obstacles in the flow resulting in their effect extending vertically according to estimates using linear theory. Correlation analysis showed that the dominant horizontal scale of the propagating eddies was about $2.5 \mathrm{~km}$, which gives an $e$ folding depth, $1 / k_{H} \sim 400 \mathrm{~m}$, that is in agreement with the simulation results.

The high-resolution simulation was compared with both aircraft data and the data from five different automatic weather stations. In general, the model compared rather well with the observations. A 1-h comparison between model and AWS data showed the model wind direction was too southerly at the Nei Lak Shan station (LT2), which is located in an elevated saddle. The model wind speed at Sunset Peak (LT6) was about 5-7 $\mathrm{m} \mathrm{s}^{-1}$ too slow. The variability of the model data also compared well with the observations, although the model had slightly less variance. The comparison of 
$(\epsilon / \rho)^{1 / 3}$ showed a rather intermittent field in the model as compared to the aircraft observations, which is in part attributable to differences in filtering. However, the mean values were similar to those derived from the aircraft data, where values in excess of $10 \mathrm{cgs}$ units, representing severe turbulence to commercial aircraft, were obtained in both cases. Perhaps the main systematic difference between the model and aircraft data was that the model overpredicted wind speeds in a small region over the new airport. The cause of this difference was not established.

Since the shear and turbulent structures are dominated by mechanical effects rather than wave dynamics, one cannot use the present results to explain mountain-induced turbulence where wave dynamics plays the dominant role. For example, in flows in the lee of the Rocky Mountains or the Aleutian Islands, gravity-wave dynamics often dominates over mechanical turbulence effects, and the presence of gravity waves may even suppress flow separation. One should not draw analogies between the scales of the foothills orography near, say, Boulder, Colorado, and Lantau Island, even though the topography in both locations rises at about the same rate to $1-\mathrm{km}$ total relief. One of the key differences is that Lantau Island is the sole source of terrain-induced turbulence in its lee, whereas the Rocky Mountain Divide, which is removed by about $25 \mathrm{~km}$ to the west of the foothills, is currently believed the main source of turbulence near Boulder during wind storm events. However, a better understanding of the role of the local terrain on the Front Range deserves further investigation. The type of problems where the current results seem most applicable are to similar small obstacles such as Ailsa Craig and the Isle of Aran in Scotland, as well as the local terrain northwest of the Bradley International Airport at Windsor Lock, Connecticut, which has reported what appears to be severe terrain-induced turbulence.

The present results suggest that the parameterization of this type of turbulence is not likely to be captured by techniques such as stochastic backscatter or buoyancy excitation, which are probably more applicable to channel shear flows dominated by aerodynamic roughness effects. In the present situation, where the turbulence appears dominated by terrain effects, the directional gradients of the orography and resulting distortion effects on the flow need to be taken into account. This appears to be a significant challenge for future research.

Our plans for future research on this topic include extending the analysis of the present simulations to better understand the nature of the instability giving rise to the transient eddies. It is still unclear as to the relative roles of the adverse pressure gradient and horizontal shearing instabilities resulting from the distorted flow. Effects of moisture will also be considered.

Acknowledgments. This research is sponsored by the Royal Observatory of Hong Kong and fulfills part of
Agreement ROC IV 7-92 VI. Per subsection 12.3 of the Agreement, findings may only be distributed for scientific, academic, and research purposes. Any other use must be authorized by the authors and the Royal Observatory of Hong Kong. The particular formulation of the stochastic backscatter was suggested to us by Alex Praskowski. The authors also wish to thank the SGI Corporation for a loan of their Power Challenge computer, which was used for the high-resolution experiment. We also thank William Anderson for his support in porting the code to the SGI machine.

\section{REFERENCES}

Arakawa, A., 1966: Computational design for long term integration of the equations of fluid motion: Two-dimensional incompressible flow. Part I. J. Comput. Phys., 1, 119-143.

Ayotte, K. W., and P. A. Taylor, 1995: A mixed spectral finite-difference 3D model of neutral planetary boundary-layer flow over topography. J. Atmos. Sci., 52, 3523-3537.

— D. Xu, and P. A. Taylor, 1994: The impact of turbulence closure schemes on predictions of the mixed spectral finite-difference model for flow over topography. Bound.-Layer Meteor., 68, 133.

Bacmeister, J. T., and R. T. Pierrehumbert, 1988: On high-drag state of nonlinear stratified flow over an obstacle. J. Atmos. Sci., 45, 63-80.

Baines, P. G., 1979: Observations of stratified flow past three-dimensional barriers. J. Geophys. Res., 84, 7834-7838.

_ , and P. C. Manins, 1989: The principles of laboratory modeling of stratified atmospheric flows over complex terrain. J. Appl. Meteor., 28, 1213-1225.

Blackadar, A. K., 1962: The vertical distribution of wind and turbulent exchange in a neutral atmosphere. J. Geophys. Res., 67, 30953102.

Castro, I. P., W. H. Snyder, and G. L. Marsh, 1983: Stratified flow over three-dimensional ridges. J. Fluid Mech., 135, 261-282.

Clark, T. L., 1977: A small scale numerical model using a terrain following coordinate transformation. J. Comput. Phys., 24, 186215 .

, and R. D. Farley, 1984: Severe downslope windstorm calculations in two and three spatial dimensions using anelastic interactive grid nesting: A possible mechanism for gustiness. $J$. Atmos. Sci., 41, 329-350.

- and W. R. Peltier, 1977: On the evolution and stability of finiteamplitude mountain waves. J. Atmos. Sci., 34, 1715-1730.

, and - 1984: Critical level reflection and the resonant growth of nonlinear mountain waves. J. Atmos. Sci., 41, 3122-3134.

, and W. D. Hall, 1991: Multi-domain simulations of the time dependent Navier-Stokes equation: Benchmark error analysis of some nesting procedures. J. Comput. Phys., 92, 456-481.

- and - 1996: On the design of smooth, conservative vertical grids for interactive grid nesting with vertical stretching. J. Appl. Meteor., 35, 1040-1046.

_ _ T. Hauf, and J. J. Kuettner, 1986: Convectively forced internal gravity waves: Results from two-dimensional numerical experiments. Quart. J. Roy. Meteor. Soc., 112, 899-925.

Durran, D. R., and J. B. Klemp, 1983: A compressible model for the simulation of moist mountain waves. Mon. Wea. Rev., 111, 2341-2361.

Finnigan, J. J., 1988: Air flow over complex terrain. Flow and Transport in the Natural Environment: Advances and Applications, W. L. Steffen and O. T. Denmead, Eds., Springer-Verlag, 183229.

Grabowski, W. W., and T. L. Clark, 1991: Cloud-environment interface instability: Rising thermal calculations in two spatial dimensions. J. Atmos. Sci., 48, 527-546. 
Hunt, J. C. R., and W. H. Snyder, 1980: Experiments on stably and neutrally stratified flow over a model three-dimensional hill. $J$. Fluid Mech., 96, 671-704.

Jackson, P. S., and J. C. R. Hunt, 1975: Turbulent wind flow over a low hill. Quart. J. Roy. Meteor. Soc., 101, 929-955.

Jenkins, G. J., P. J. Mason, W. H. Moores, and R. I. Sykes, 1981: Measurements of the flow structure around Alisa Craig, a steep, three-dimensional, isolated hill. Quart. J. Roy. Meteor. Soc., 107, 833-851.

Klemp, J. B., and D. K. Lilly, 1978: Numerical simulation of hydrostatic mountain waves. J. Atmos. Sci., 35, 78-107.

Laprise, R., and W. R. Peltier, 1989: The linear stability of nonlinear mountain waves: Implications for the understanding of severe downslope windstorms. J. Atmos. Sci., 46, 545-564.

Leith, C. E., 1990: Stochastic backscatter in a subgrid-scale model: Plane shear mixing layer. Phys. Fluids A, 2, 297-299.

Lilly, D. K., 1962: On the numerical simulation of buoyant convection. Tellus, 14, 145-172.

— 1972-A meteorological narrative. Weatherwise, 25, 56-63.

_, and J. B. Klemp, 1979: The effects of terrain shape on nonlinear hydrostatic mountain waves. J. Fluid Mech., 95, 241-261.

Lipps, F. G., and R. S. Hemler, 1982: A scale analysis of deep moist convection and some related numerical calculations. J. Atmos. Sci., 39, 2192-2210.

Mason, P. J., 1986: Flow over the summit of an isolated hill. Bound.Layer Meteor., 37, 385-405.

- and R. I. Sykes, 1979: Flow over an isolated hill of moderate slope. Quart. J. Roy. Meteor. Soc., 105, 383-395.

—, and J. C. King, 1985: Measurements and predictions of flow and turbulence over an isolated hill of moderate slope. Quart. J. Roy. Meteor. Soc., 111, 617-640.

— simulations of boundary layers. J. Fluid Mech., 242, 51-78.
Peltier, W. R., and T. L. Clark, 1979: The evolution and stability of finite-amplitude mountain waves. Part II: Surface wave drag and severe downslope windstorms. J. Atmos. Sci., 36, 1489-1529.

- , and -1983 : Non-linear mountain waves in two and three spatial dimensions. Quart. J. Roy. Meteor. Soc., 109, 527-548.

Reisner, J. M., and P. K. Smolarkiewicz, 1994: Thermally forced low froude number flow past three-dimensional obstacles. J. Atmos. Sci., 51, 117-133.

Romero, R., S. Alonso, E. C. Nickerson, and C. Ramis, 1995: The influence of vegetation on the development and structure of mountain waves. J. Appl. Meteor., 34, 2230-2242.

Savill, A. M., 1987: Recent developments in rapid-distortion theory. J. Fluid Mech., 19, 531-575.

Schlichting, H., 1960: Boundary-Layer Theory. McGraw-Hill, 647 pp

Scinocca, J. F., and W. R. Peltier, 1991: On the Richardson number dependence of nonlinear critical-layer flow over localized topography. J. Atmos. Sci., 48, 1560-1572.

Smagorinsky, J., 1963: General circulation experiments with the primitive equations. Proc. Int. Symp. on Numerical Weather Prediction, Tokyo, Japan, Meteor. Soc. Japan, 85-107.

Smith, R. B., A. C. Gleason, P. A. Gluhosky, and V. Grubisic, 1997: The wake of St. Vincent. J. Atmos. Sci., 54, 606-623.

Smolarkiewicz, P. K., 1983: A simple positive definite advection scheme with small implicit diffusion. Mon. Wea. Rev., 111, 479486.

, 1984: A fully multidimensional positive definite advection transport algorithm with small implicit diffusion. J. Comput. Phys., 54, 325-362.

Xu, D., K. W. Ayotte, and P. A. Taylor, 1994: Development of a nonlinear mixed spectral finite difference model for turbulent boundary-layer flow over topography. Bound.-Layer Meteor., 70, 341367. 